\title{
ON A MODIFIED PARABOLIC COMPLEX MONGE-AMPÈRE EQUATION WITH APPLICATIONS
}

\author{
ALBERT CHAU ${ }^{1}$ AND LUEN-FAI TAM ${ }^{2}$
}

\begin{abstract}
We study a parabolic complex Monge-Ampère type equation of the form (1.1) on a complete non-compact Kähler manifold. We prove a short time existence result and obtain basic estimates. Applying these results, we prove that under certain assumptions on a given real and closed $(1,1)$ form $\Omega$ and initial Kähler metric $g_{0}$ on $M$, the modified Kähler-Ricci flow $g^{\prime}=-$ Ric $+\Omega$ has a long time solution converging to a complete Kähler metric such that Ric $=\Omega$, which extends the result in [1] to non-compact manifolds. We will also obtain a long time existence result for the Kähler-Ricci flow which generalizes a result [5].
\end{abstract}

\section{INTRODUCTION}

Let $\left(M, g_{0}\right)$ be a smooth complete non-compact Kähler manifold. In this article we will study parabolic complex-Monge Ampère equations of the following type on $M$ :

$$
\left\{\begin{array}{l}
\frac{\partial v}{\partial t}=\log \frac{(\sigma(t)+\sqrt{-1} \partial \bar{\partial} v)^{n}}{\left(\omega_{0}\right)^{n}}-f \text { in } M \times[0, T) \\
v(x, 0)=u
\end{array}\right.
$$

where $f$ and $\sigma(t)$ are given smooth families of functions and real and closed $(1,1)$ forms on $M$ for $t \in[0, T)$ respectively, $u$ is a smooth function on $M$ and $\omega_{0}$ is the Kähler form of $g_{0}$. There is a close connection between (1.1) and the Kähler-Ricci flow equation on $M$

$$
\left\{\begin{array}{l}
\frac{\partial \omega}{\partial t}=-\operatorname{Ric}(\omega) \\
\omega(0)=\omega_{0}
\end{array}\right.
$$

\footnotetext{
${ }^{1}$ Research partially supported by NSERC grant no. \#327637-06.

${ }^{2}$ Research partially supported by Hong Kong RGC General Research Fund \#GRF 2160357.

2000 Mathematics Subject Classification. Primary 53C55, 58J35.

Key words and phrases. Non-compact Kähler-Einstein metrics, Kähler-Ricci flow, parabolic Monge-Ampère equation.
} 
where $\omega$ is a Kähler form with corresponding Ricci form $\operatorname{Ric}(\omega)$. Let $v$ be a solution to (1.1) such that the corresponding family $\omega(t):=\sigma(t)+$ $\sqrt{-1} \partial \bar{\partial} v$ are Kähler forms. Then if $\operatorname{Ric}_{0}=\sqrt{-1} \partial \bar{\partial} f$ and $\sigma(t)=\omega_{0}$, then $\omega(t)$ evolves under (1.2) and remains in the Kähler class $\left[\omega_{0}\right]$ (see [1], [6]). On the other hand, if $f=0$ and $\sigma_{t}=-t \operatorname{Ric}\left(\omega_{0}\right)+\omega_{0}$, then $\omega(t)$ evolves under (1.2) but does not remain in the same Kähler class in general (see [20, 21] on compact manifolds and [1] on non-compact manifolds). Conversely, it can also be shown in the above cases that given a solution $\omega(t)$ to (1.2), there exists a corresponding solution $v$ to (1.1) such that $\omega(t):=\sigma(t)+\sqrt{-1} \partial \bar{\partial} v$ (see references above).

One of the goals in this article is to generalize our previous results in [6] where the authors proved: when $\operatorname{Ric}_{0}=\sqrt{-1} \partial \bar{\partial} f$ and $\sigma(t)=\omega_{0}$, then $g_{0}$ converges to a Kähler Ricci flat metric $g$ under (1.2) under certain additional assumptions on $f$ and $g_{0}$. We will extend this result to the case when $\operatorname{Ric}_{0}-\Omega=\sqrt{-1} \partial \bar{\partial} f$ where $\Omega$ is given but not necessarily zero. We prove that when $\left(M^{n}, g_{0}\right)$ is complete, non-compact with bounded curvature, with volume growth $V_{x_{0}}(r) \leq \mathrm{Cr}^{2 n}$ for some $x_{0}$ and $C$ for all $r$, and satisfies a certain Sobolev inequality, then:

Under the above conditions, the Kähler-Ricci flow (1.2) has a long time solution $g(t)$ converging smoothly on $M$ provided $|f|(x) \leq \frac{C}{1+\rho_{0}^{2+\epsilon}(x)}$ for some $C, \epsilon>0$ and all $x$ such that the Ricci form of the limit metric is $\Omega$.

See Theorem 4.2 for details. This will correspond to (1.1) in the case $\omega(t)=\omega_{0}$ and $\operatorname{Ric}_{0}-\Omega=\sqrt{-1} \partial \bar{\partial} f$ in which case the equation for $\frac{\partial}{\partial t} \omega(t)$ will change from (1.2) only by the addition of $\Omega=\sqrt{-1} \partial \bar{\partial} f$ to the RHS of (1.2). The proof combines the a priori estimates developed here together with estimates from [6], in particular the $C^{0}$ estimate. The main difference here is that the corresponding metrics $g(t)$ are not evolving under the standard Kähler-Ricci flow (1.2), and general Kähler-Ricci theory cannot be directly applied as in [6]. Our results are motivated by the results in [18, 19] which extend the famous results in [22] to the complete non-compact setting under additional assumptions. By studying the elliptic Monge-Ampère equation Yau [22] proved that if $\left(M, g_{0}\right)$ is a compact Kähler manifold and $\Omega \in c_{1}(M)$, then there exists a Kähler metric $g$ in the same class as $g_{0}$ with $\Omega$ as its Ricci tensor. This result was later re-established in [1] by considering the corresponding parabolic complex Monge-Ampère on compact manifolds.

Our second goal will be to establish a longtime existence result for (1.2). We prove that when $\left(M^{n}, g_{0}\right)$ is smooth, complete and non-compact with injectivity radius bounded below and curvature approaching zero pointwise at infinity then 
Under the above conditions, the Kähler-Ricci flow (1.2) has a smooth longtime solution provided there exists a strictly plurisubharmonic function on $M$.

See Corollary 4.1 for details. Since a simply connected complete noncompact Kähler manifold with nonnegative holomorphic bisectional curvature is a product of a compact Kähler manifold with nonnegative holomorphic bisectional curvature and a complete non-compact Kähler manifold with nonnegative holomorphic bisectional curvature supporting a strictly pluri-subharmonic function [13], the theorem generalizes the longtime existence result in [5]. In particular, the result applies when $M=\mathbb{C}^{n}$ or more generally a Stein manifold. We establish this by showing (1.1) has a longtime solution for appropriate choices of $f$ and $\sigma(t)$. The proof combines ideas from [11], the a priori estimates developed here and the results in [5]. In fact, this is a corollary of a more general result Theorem 4.1.

The paper is organized roughly as follows. In $\S 2$ we prove a general shorttime existence result for (1.1) where we do not assume $f$ or $v(x, 0)$ are bounded. In $\S 3$ we prove a priori estimates for (1.1) where we assume $f$ and $v(x, 0)=0$ are bounded. In $\S 4$ we prove the main results Theorem 4.1 and Theorem 4.2 .

\section{Short time Existence}

Consider parabolic complex Monge-Ampère equation (1.1) on a complete non-compact Kähler manifold $\left(M, g_{0}\right)$ where $\sigma=\sigma(t)$ is a given smooth real and closed $(1,1)$ form on $M$ for $t \in[0, T), \omega_{0}$ is the Kähler form of $g_{0}$, and $f$ and $u$ are smooth possibly unbounded functions on $M \times[0, T)$ and $M$ respectively.

We will do our analysis of (1.1) in appropriate Hölder spaces on $M$ which in turn will involve the notions of bounded geometry of various orders with respect to $g_{0}$. We will now make the appropriate definitions for these. We begin by recalling the definition for a complete Kähler manifold $\left(M^{n}, g\right)$ ( $n$ is the complex dimension) to have bounded geometry of a certain order, and we also recall the corresponding parabolic and elliptic Hölder spaces on $M$ relative to $g_{0}$ (see also [2, 18, 19]).

Definition 2.1. Let $\left(M^{n}, g\right)$ complete Kähler manifold. Let $k \geq 1$ be an integer and $0<\alpha<1 . g$ is said to have bounded geometry of order $k+\alpha$ if there are positive numbers $r, \kappa_{1}, \kappa_{2}$ such that at every $p \in M$ there is a neighborhood $U_{p}$ of $p$, and local biholomorphism $\xi_{p}$ from $D(r)$ onto $U_{p}$ with $\xi_{p}(0)=p$ satisfying the following properties: 
(i) the pull back metric $\xi_{p}^{*}(g)$ satisfies:

$$
\kappa_{1} g_{e} \leq \xi_{p}^{*}(g) \leq \kappa_{2} g_{e}
$$

where $g_{e}$ is the standard metric on $\mathbb{C}^{n}$;

(ii) the components $g_{i \bar{\jmath}}$ of $\xi_{p}^{*}(g)$ in the natural coordinate of $D(r) \subset$ $\mathbb{C}^{n}$ are uniformly bounded in the standard $C^{k+\alpha}$ norm in $D(r)$ independent of $p$.

It is obvious that if $g_{0}$ is of bounded geometry of order $k+\alpha$, then it is of bounded geometry of order $k+\alpha^{\prime}$ for all $\alpha^{\prime}<\alpha$. The family

$$
\mathcal{F}=\left\{\left(\xi_{p}, U_{p}\right), p \in M\right\}
$$

is called a family of quasi-coordinate neighborhoods.

In the following we will define a norm on $m$ forms on $M$ for any $m$ and we will define corresponding Banach spaces. We will make these definitions relative to fixed quasi-coordinates $\mathcal{F}$. For two different quasi-coordinates, the corresponding norms defined will be equivalent $\mathrm{f}$, and the corresponding Banach spaces will be the same. See the appendix for details.

For any domain $\Omega$ in $\mathbb{C}^{n}$ and integer $k \geq 0$ and $0<\alpha<1$, let $\|\cdot\|_{\Omega, k+\alpha}$ be the standard $C^{k+\alpha}$ norm for functions on $\Omega$. If $T>0$ and $k$ is even let $\|\cdot\|_{\Omega \times[0, T) ; k+\alpha, k / 2+\alpha / 2}$ be the standard parabolic $C^{k+\alpha, k / 2+\alpha / 2}$ norm for functions on $\Omega \times[0, T)$ (see appendix see details).

Define the $C^{k+\alpha}(M)$ norm for a smooth $m$-form $f$ on $M$ by

$$
\|f\|_{m, k, \alpha}=\sup _{p \in M} \max _{I}\left\|\left(\xi_{p}^{*} f\right)_{I}\right\|_{D(r), k+\alpha} .
$$

where $I$ represents a multi-index and the $\left(\xi_{p}^{*} f\right)_{I}$ 's are the local components of the form $\xi_{p}^{*} f$. Likewise, if $T>0$ and $k$ is even define the $C^{k+\alpha, k / 2+\alpha / 2}(M \times[0, T))$ norm for smooth time dependent $m$-form $f$ on $M \times[0, T)$ by

$$
\|f\|_{m, k, \alpha}=\sup _{p \in M} \max _{I}\left\|\left(\xi_{p}^{*} f\right)_{I}\right\|_{D(r) \times[0, T) ; k+\alpha, k / 2+\alpha / 2} .
$$

Definition 2.2. For any $m$ and $0 \leq k \leq 2$ we define $C_{m}^{k+\alpha}(M)$ to be the norm completion of space of smooth $m$-forms with norm $\|\cdot\|_{m, k, \alpha}$. Likewise, for $k=0$ or 2 , let $C_{m}^{k+\alpha, k / 2+\alpha / 2}(M \times[0, T))$ be the norm completion of space of smooth time dependent $m$-forms with norm $\|\cdot\|_{m, k, \alpha}$. Both $C_{m}^{k+\alpha}(M)$ and $C_{m}^{k+\alpha, k / 2+\alpha / 2}(M \times[0, T))$ are Banach spaces. We will omit the subscript $m$ in the notation when there will be no confusion.

We have the following lemma: 2.1, 
Lemma 2.1. Let $\left(M^{n}, g\right)$ be a complete non-compact Kähler manifold of bounded geometry of order $2+\alpha$. Then

(i) $M$ has bounded curvature.

(ii) There is a smooth function $\rho \geq 1$ such that near infinity it is equivalent to the distance function from a fixed point and has bounded gradient and Hessian.

Proof. (i) is obvious from the definition of bounded geometry of order $2+\alpha$. (ii) is a result in [15], see also [16].

In order to state the main result of short time existence of (1.1), we first discuss the following special case:

$$
\left\{\begin{array}{l}
\frac{\partial v}{\partial t}=\log \frac{(\sigma(t)+\sqrt{-1} \partial \bar{\partial} v)^{n}}{\left(\omega_{0}\right)^{n}} \\
v(x, 0)=0 .
\end{array}\right.
$$

Lemma 2.2. Let $\left(M^{n}, g_{0}\right)$ be a smooth complete non-compact Kähler manifold of bounded geometry of order $2+\alpha$ and let $\sigma=\sigma(t)$ be a smooth family of closed and real $(1,1)$ forms on $M \times[0, T)$ such that

(i) $\sigma \in C^{\alpha, \frac{\alpha}{2}}(M \times[0, T))$;

(ii) $c^{-1} \omega_{0} \leq \sigma \leq c \omega_{0}$ for some $c>0$ on $M \times[0, T)$;

(iii) there exists $v_{0} \in C^{2+\alpha, 1+\frac{\alpha}{2}}(M \times[0, T))$ such that

$$
w_{0}:=\frac{\partial v_{0}}{\partial t}-\log \left(\frac{\left(\sigma+\sqrt{-1} \partial \bar{\partial} v_{0}\right)^{n}}{\omega_{0}^{n}}\right)
$$

satisfies $w_{0}(x, 0)=0$.

Then there exists $0<T^{\prime} \leq T$ such that (2.3) has a smooth solution $v \in C^{2+\frac{\alpha}{2}, 1+\frac{\alpha}{4}}\left(M \times\left[0, T^{\prime}\right]\right)$ so that $\sigma+\sqrt{-1} \partial \bar{\partial} v$ is uniformly equivalent to $\omega_{0}$ in $M \times\left[0, T^{\prime}\right]$.

Proof. The idea is based on a general implicit function Theorem argument outlined in [9] 1. By choosing a smaller $T$ if necessary, we may assume that $\sigma$ is uniformly equivalent to $\omega_{0}$ in $M \times[0, T]$. In particular, there exists $\delta>0$ and $C_{1}>0$ such that if $\|\mid v\|_{2+\frac{\alpha}{2}, 1+\frac{\alpha}{4}}<\delta$ then $C_{1} \omega_{0} \geq \sigma+\sqrt{-1} \partial \bar{\partial} v \geq C_{1}^{-1} \omega_{0}$ in $M \times[0, T]$. For the remainder of the proof for any $k$ and $\beta$ we will denote the spaces $C^{k+\beta, k / 2+\beta / 2}(M \times[0, T])$ and $C^{k+\beta}(M)$ simply by $C^{k+\beta, k / 2+\beta / 2}$ and $C^{k+\beta}$.

We define

$$
\mathcal{B}=\left\{v \in C^{2+\frac{\alpha}{2}, 1+\frac{\alpha}{4}} \mid\|v\|_{2+\frac{\alpha}{2}, 1+\frac{\alpha}{4}}<\delta, v(x, 0)=0\right\}
$$

\footnotetext{
${ }^{1}$ Also see Proposition 5.1 in 3 for an application of this argument to the entire graphical Mean Curvature flow
} 
Then $\mathcal{B}$ is an open ball in a closed subspace of $C^{2+\frac{\alpha}{2}, 1+\frac{\alpha}{4}}$. Now define the map

$$
F: \mathcal{B} \rightarrow C^{\frac{\alpha}{2}, \frac{\alpha}{4}}
$$

by

$$
F(v)=\frac{\partial v}{\partial t}-\log \frac{(\sigma+\sqrt{-1} \partial \bar{\partial} v)^{n}}{\omega^{n}} .
$$

Then the map $F$ is well defined and $C^{1}$ such that the differential $D F_{v}$ at any $v \in \mathcal{B}$ is given by

$$
D F_{v}(\phi)=\frac{\partial \phi}{\partial t}-\left({ }^{v} \sigma\right)^{i \bar{\jmath}} \phi_{i \bar{\jmath}}
$$

where $\left({ }^{v} \sigma\right)^{i \bar{\jmath}}$ is the inverse of $\left({ }^{v} \sigma\right)_{i \bar{\jmath}}:=\sigma_{i \bar{\jmath}}+v_{i \bar{\jmath}}$.

Claim 1: $D F_{v}$ is a bijection from the Banach space

$$
\mathcal{B}_{1}=\left\{\phi \in C^{2+\frac{\alpha}{2}, 1+\frac{\alpha}{4}} \mid \phi(x, 0)=0\right\}
$$

onto $C^{\frac{\alpha}{2}, \frac{\alpha}{4}}$.

Note that the claim is straight forward in the case that ${ }^{v} \sigma$ has bounded curvature on $M \times[0, T]$. As we cannot assume this however, we must proceed more carefully. Let $\rho$ be a smooth function on $M$ equivalent to the distance function with respect to $g_{0}$ from some point $p$ as Lemma 3. Since the metrics ${ }^{v} \sigma$ are uniformly equivalent to $g_{0}$ and $\rho \geq 1$, there is a constant $C_{2}$ such that

$$
\left|{ }^{v} \sigma_{i \bar{\jmath}} \rho_{i \bar{\jmath}}\right|<C_{2} \rho
$$

in $M \times[0, T]$. Hence if $\phi \in \mathcal{B}_{1}$, then for any $\epsilon>0$,

$$
\frac{\partial}{\partial t}\left(\phi+\epsilon e^{C_{2} t} \rho\right)-{ }^{v} \sigma_{i \bar{\jmath}}\left(\phi_{i \bar{\jmath}}+\epsilon e^{C_{2} t} \rho_{i \bar{\jmath}}\right)>0 .
$$

On the other hand, the minimum of $\phi+\epsilon e^{C_{2} t} \rho$ is attained in a compact set of $M \times[0, T]$, and thus by the maximum principle, we conclude that $\phi+\epsilon e^{C_{2} t} \rho \geq 0$ because $\phi(x, 0)=0$. Letting $\epsilon \rightarrow 0$, we conclude $\phi \geq 0$ on $M \times[0, T]$. Similarly, one can prove that $\phi \leq 0$. Hence $\phi=0$ and $D F_{v}$ is injective.

Now, let $\Omega_{l}$ be bounded domains with smooth boundary which exhaust $M$ and ${ }^{l} \phi$ be the solution of $D F_{v}\left({ }^{l} \phi\right)=w$ in $\Omega_{l} \times[0, T]$ where $\phi^{l}=0$ for $t=0$ and on $\partial \Omega_{l} \times[0, T]$ and $w \in C^{\frac{\alpha}{2}, \frac{\alpha}{4}}(M \times[0, T]$. Let $C_{3}>\sup _{M \times[0, T]}|w|$. Then $D F_{v}\left({ }^{l} \phi+C_{3} t\right)>0$. By the maximum principle, we conclude that ${ }^{l} \phi \geq-C_{3}$. Similarly, we have ${ }^{l} \phi \leq C_{3}$. Hence the sequence $\left.\right|^{l} \phi \mid$ is uniformly bounded by $C_{3}$. 
Now for any $p \in M$, let $\left(\xi_{p}, U_{p}\right)$, and $\xi_{p}: D(r) \rightarrow U_{p}$ be as in Definition 2.1. The pull back of ${ }^{l} \phi$ satisfies:

$$
\frac{\partial^{l} \phi}{\partial t}-\left({ }^{v} \sigma\right)^{i \bar{\jmath}}\left({ }^{l} \phi\right)_{i \bar{\jmath}}=w
$$

in $D(r)$. For simplicity, we use ${ }^{l} \phi$ to denote the pull back of ${ }^{l} \phi$, etc. By our hypothesis, the components $\left({ }^{v} \sigma\right)^{i \bar{\jmath}}$ above are uniformly equivalent to the standard Euclidean metric and are uniformly bounded in the standard $C^{\alpha, \frac{\alpha}{2}}$ norm on $D(r) \times[0, T]$. Then by standard Schauder estimates we have

$$
\|\|^{l} \phi \|_{D\left(\frac{r}{2}\right) \times[0, T], 2+\frac{\alpha}{2}, 1+\frac{\alpha}{4}} \leq C_{4}
$$

for some $C_{4}$ independent of $p$ and sufficiently large $l$. Now a standard diagonalizing subsequence argument produces a $\phi \in C^{2+\frac{\alpha}{2}, 1+\frac{\alpha}{4}}$ such that $D F_{v}(\phi)=w$. So $D F_{v}$ is surjective and the claim is established.

Now let $v_{0}$ be the function in (iii) which is in $C^{2+\alpha, 1+\frac{\alpha}{2}}(M \times[0, T)$, then $w_{0}=F\left(v_{0}\right)$ and $w_{0}(x, 0)=0$. By the inverse function theorem, there exists $\epsilon>0$ such that if $\left\|w-w_{0}\right\|_{\frac{\alpha}{2}, \frac{\alpha}{4}}<\epsilon$, then there is $v \in$ $C^{2+\frac{\alpha}{2}, 1+\frac{\alpha}{4}}$ such that $F(v)=w$.

For any $0<\tau<1$, let $w_{\tau}$ be such that

$$
w_{\tau}(x, t)= \begin{cases}0, & t \leq \tau \\ w_{0}(x, t-\tau), & \tau<t<1\end{cases}
$$

Claim 2: $\left\|w_{\tau}-w_{0}\right\|_{\frac{\alpha}{2}}, \frac{\alpha}{4}<\epsilon$ for sufficiently small $\tau>0$.

We will still use $w_{\tau}$ and $w_{0}$ to denote the respective pull backs under $\xi_{p}$. Let $x, x^{\prime} \in D(r), t, t^{\prime}$. Let $A$ be the $2+\alpha$ norm of $w_{0}$ and let $\eta=w_{\tau}-w_{0}$.

Case 1: $t, t^{\prime} \leq \tau$. Then

$$
\begin{aligned}
\left|\eta(x, t)-\eta\left(x^{\prime}, t^{\prime}\right)\right| & =\left|w_{0}(x, t)-w_{0}\left(x^{\prime}, t^{\prime}\right)\right| \\
& \leq A \min \left\{\left(\left|x-x^{\prime}\right|^{\alpha}+\left|t-t^{\prime}\right|^{\frac{\alpha}{2}}\right), t^{\frac{\alpha}{2}}+\left(t^{\prime}\right)^{\frac{\alpha}{2}}\right\} .
\end{aligned}
$$

where we have used the fact that $\left.w_{0}\right|_{t=0}=0$. Thus if $\left|x-x^{\prime}\right| \geq \tau^{\frac{1}{2}}$ then $\left|\eta(x, t)-\eta\left(x^{\prime}, t^{\prime}\right)\right| \leq A\left(t^{\frac{\alpha}{2}}+\left(t^{\prime}\right)^{\frac{\alpha}{2}}\right) \leq 2 A \tau^{\frac{\alpha}{2}} \leq 2 A \tau^{\frac{\alpha}{4}}\left(\left|x-x^{\prime}\right|^{\frac{\alpha}{2}}+\left|t-t^{\prime}\right|^{\frac{\alpha}{4}}\right)$, and if $\left|x-x^{\prime}\right| \leq \tau^{\frac{1}{2}}$ then $\left|\eta(x, t)-\eta\left(x^{\prime}, t^{\prime}\right)\right| \leq A\left(\left|x-x^{\prime}\right|^{\alpha}+\left|t-t^{\prime}\right|^{\frac{\alpha}{2}}\right) \leq A \tau^{\frac{\alpha}{4}}\left(\left|x-x^{\prime}\right|^{\frac{\alpha}{2}}+\left|t-t^{\prime}\right|^{\frac{\alpha}{4}}\right)$ because $\left|t-t^{\prime}\right| \leq \tau$. In either case above we see that the claim is true.

Case 2: $t, t^{\prime} \geq \tau$. Then 


$$
\begin{aligned}
\left|\eta(x, t)-\eta\left(x^{\prime}, t^{\prime}\right)\right| & =\left|w_{0}(x, t)-w_{0}\left(x^{\prime}, t^{\prime}\right)-w_{0}(x, t-\tau)+w_{0}\left(x^{\prime}, t^{\prime}-\tau\right)\right| \\
& \leq 2 A \min \left\{\tau^{\frac{\alpha}{2}},\left|x-x^{\prime}\right|^{\alpha}+\left|t-t^{\prime}\right|^{\frac{\alpha}{2}}\right\}
\end{aligned}
$$

Thus if $\left|t-t^{\prime}\right| \geq \tau$, then

$$
\left|\eta(x, t)-\eta\left(x^{\prime}, t^{\prime}\right)\right| \leq 2 A \tau^{\frac{\alpha}{2}} \leq 2 A \tau^{\frac{\alpha}{4}}\left(\left|x-x^{\prime}\right|^{\frac{\alpha}{2}}+\left|t-t^{\prime}\right|^{\frac{\alpha}{4}}\right),
$$

and if $\left|t-t^{\prime}\right| \leq \tau$, then we can prove as in Case 1 that

$$
\left|\eta(x, t)-\eta\left(x^{\prime}, t^{\prime}\right)\right| \leq 2 A \tau^{\frac{\alpha}{4}}\left(\left|x-x^{\prime}\right|^{\frac{\alpha}{2}}+\left|t-t^{\prime}\right|^{\frac{\alpha}{4}}\right) .
$$

In either case above we see that the claim is true.

Hence by the inverse function theorem we have $F(v)=0$ on $M \times[0, \tau]$ for sufficiently small $\tau$. In particular $v$ solves (2.3) and satisfies the conditions in the lemma $M \times[0, \tau]$. The fact that $v$ is smooth follows from a standard bootstrapping argument applied to (2.3) as at the end of the proof of Proposition (2.1).

Proposition 2.1. Let $\left(M^{n}, g_{0}\right)$ be a smooth complete non-compact Kähler manifold of bounded geometry of order $2+\alpha$. Let $u$ be a smooth function on $M$ and let $f$ and $\sigma=\sigma(t)$ be a smooth family of functions and real and closed $(1,1)$ forms, respectively, on $M$ for $t \in[0, T)$. Suppose that

(i) $\left(\|d u\|_{C^{1+\alpha}(M)}+\left|f_{t}\right|+\sup _{t \in[0, T)}\|d f(t)\|_{C^{1+\alpha}(M)}\right)<\infty$;

(ii) $\sigma \in C^{2+\alpha, 1+\frac{\alpha}{2}}(M \times[0, T))$;

(iii) $\sigma+\sqrt{-1} \partial \bar{\partial} u \geq c \omega_{0}$ on $M \times[0, T)$ for some $c>0$;

(iv) $\log \frac{(\sigma+\partial \bar{\partial} u)^{n}}{\omega_{0}^{n}}$ is in $C^{2+\alpha, 1+\frac{\alpha}{2}}(M \times[0, T))$.

Then there exists $0<T^{\prime} \leq T$ and a smooth solution $v$ to (1.1) on $M \times\left[0, T^{\prime}\right]$ such that $\left(v-u+\int_{0}^{t} f(s) d s\right) \in C^{2+\alpha, 1+\alpha / 2}\left(M \times\left[0, T^{\prime}\right]\right)$ and $\sigma+\sqrt{-1} \partial \bar{\partial} v$ are uniformly equivalent to $\omega_{0}$ in $M \times\left[0, T^{\prime}\right]$.

Proof. Let $u, \sigma$ and $f$ be as in the Proposition and let

$$
\tilde{\sigma}_{i \bar{\jmath}}=\sigma_{i \bar{\jmath}}+\left(u-\int_{0}^{t} f(s) d s\right)_{i \bar{\jmath}} .
$$

Then $\tilde{\sigma} \in C^{\alpha, \frac{\alpha}{2}}\left(M \times\left[0, T^{\prime}\right]\right)$ and there is $T^{\prime} \in(0, T)$ and $c>0$ such that

$$
c \omega_{0} \geq \tilde{\sigma} \geq c \omega_{0}
$$


in $M \times\left[0, T^{\prime}\right]$. Let

$$
v_{0}=t \log \left(\frac{(\tilde{\sigma}+\sqrt{-1} \partial \bar{\partial} u)^{n}}{\omega_{0}^{n}}\right)
$$

Then $v_{0}$ satisfies condition (iii) in Lemma 2.2 with $\sigma$ replaced by $\tilde{\sigma}$. By Lemma 2.2, by choose a smaller $T^{\prime}$ we may assume that there is $\tilde{v} \in C^{2+\frac{\alpha}{2}, 1+\frac{\alpha}{4}}\left(M \times\left[0, T^{\prime}\right]\right.$ solving (2.3) (with $\sigma$ replaced by $\tilde{\sigma}$ ) such that $\tilde{\sigma}+\sqrt{-1} \partial \bar{\partial} v$ is uniformly equivalent to $\omega_{0}$ in $M \times\left[0, T^{\prime}\right]$.

Let $v=\tilde{v}+u-\int_{0}^{t} f(s) d s$. Then

$\sigma+\sqrt{-1} \partial \bar{\partial} v=\sigma+\sqrt{-1} \partial \bar{\partial} \tilde{v}+\sqrt{-1} \partial \bar{\partial}\left(u-\int_{0}^{t} f(s) d s\right)=\tilde{\sigma}+\sqrt{-1} \partial \bar{\partial} \tilde{v}$

and

$$
\frac{\partial v}{\partial t}=\frac{\partial \tilde{v}}{\partial t}-f
$$

Hence $v$ is a solution to (1.1), $v$ is smooth and $\sigma+\sqrt{-1} \partial \bar{\partial} v$ is uniformly equivalent to $\omega_{0}$ in $M \times\left[0, T^{\prime}\right]$. It remains to prove that $\tilde{v}$ is actually in $C^{2+\alpha, 1+\frac{\alpha}{2}}\left(M \times\left[0, T^{\prime}\right]\right)$.

Around any $p \in M$, pull the equation (1.1) back into $D(r)$ by $\xi_{p}$ and differentiate the pullback equation with respect to $z^{l}$ say. We then obtain the following in $D(r) \times[0, T]$

$$
\left\{\begin{array}{l}
\left.\frac{\partial v_{l}}{\partial t}=\left({ }^{v} \sigma\right)\right)^{i \bar{\jmath}}\left(v_{l}\right)_{i \bar{\jmath}}+\left({ }^{v} \sigma\right)^{i \bar{\jmath}}(\sigma)_{i \bar{\jmath} l}-\left(g_{0}\right)^{i \bar{\jmath}}\left(g_{0}\right)_{i \bar{\jmath} l}-f_{l} \\
v_{l}(x, 0)=u_{l} .
\end{array}\right.
$$

Thus (2.6) is a strictly parabolic equation for $v_{l}$ with initial condition $u_{l}$ being in $C^{3+\alpha}\left(D\left(\frac{3}{4} r\right)\right.$ ) (by condition (i), (ii) and (iv)) such that $\left\|u_{l}\right\|_{3+\alpha, D\left(\frac{3}{4} r\right)}$ is bounded by a constant independent of $p$. Moreover, the $C^{\frac{\alpha}{2}, \frac{\alpha}{4}}$ norms of $\left({ }^{v} \sigma\right)^{i \bar{\jmath}}$ and $\left({ }^{v} \sigma\right)^{i \bar{\jmath}}(\sigma)_{i \bar{\jmath} l}-\left(g_{0}\right)^{i \bar{\jmath}}\left(g_{0}\right)_{i \bar{\jmath} l}-f_{l}$ in $D(r) \times$ $\left[0, T^{\prime}\right]$ are bounded by a constant independent of $t$. It follows from parabolic Schauder theory that the $C^{2+\frac{\alpha}{2}, 1+\frac{\alpha}{4}}$ norm of $v_{l}$ in $(D(r / 2) \times$ $\left.\left[0, T^{\prime}\right]\right)$ is bounded by a constant independent of $p$. Repeating the above argument with respect to a conjugate coordinate $z_{\bar{l}}$, we conclude that $C^{2+\frac{\alpha}{2}, 1+\frac{\alpha}{4}}$ norms of the first space derivatives of $v$ in $D\left(\frac{r}{2}\right) \times\left[0, T^{\prime}\right]$ are bounded by a constant independent of $p$. This implies in particular, that the $C^{\alpha, \frac{\alpha}{2}}$ norms of $\left.\left({ }^{v} \sigma\right)\right)^{i \bar{\jmath}}$ and $\left({ }^{v} \sigma\right)^{i \bar{\jmath}}(\sigma)_{i \bar{\jmath} l}-\left(g_{0}\right)^{i \bar{\jmath}}\left(g_{0}\right)_{i \bar{\jmath} l}-f_{l}$ in $D\left(\frac{r}{2}\right) \times\left[0, T^{\prime}\right]$ are bounded by a constant independent of $t$ (see remark 1). Repeating the above arguments, together with the fact that $\tilde{v}$ is uniformly bounded and the assumptions on $u$ and $f$ we conclude that $\tilde{v}$ is in the $C^{2+\alpha, 1+\frac{\alpha}{2}}\left(M \times\left[0, T^{\prime}\right]\right)$. By the bootstrapping argument above it is also not hard to see that $v$ is in fact smooth. 
Remark 1 . In the second to last sentence in the proof above we actually have that the $C^{\frac{\alpha}{2}}, \frac{\alpha}{4}$ norms of the first space derivatives of $\left.\left({ }^{v} \sigma\right)\right)^{i \bar{\rho}}$ and $\left({ }^{v} \sigma\right)^{i \bar{\jmath}}(\sigma)_{i \bar{\jmath} l}-\left(g_{0}\right)^{i \bar{\jmath}}\left(g_{0}\right)_{i \bar{\jmath} l}-f_{l}$ in $D\left(\frac{r}{2}\right) \times\left[0, T^{\prime}\right]$ are bounded by a constant independent of $t$. Thus by the above argument we in fact have that the $C^{2+\frac{\alpha}{2}, 1+\frac{\alpha}{4}}$ norms of the second space derivatives of $v$ in $D\left(\frac{r}{4}\right) \times\left[0, T^{\prime}\right]$ are bounded by a constant independent of $p$.

The shorttime existence of (1.2) was proved by Shi [14, 15] assuming $\left(M^{n}, g_{0}\right)$ is a complete non-compact Kähler manifold with bounded curvature. Proposition 2.1 re-establishes this fact under the additional assumption of bounded geometry of order $2+\alpha$ such that the Ricci form is in $C^{2+\alpha}(M)$, with much shorter proof. In particular, we have

Corollary 2.1. Let $\left(M^{n}, g_{0}\right)$ be a complete non-compact Kähler manifold with bounded geometry of order $2+\alpha$ and the Ricci form is in $C^{2+\alpha}(M)$ for some $\alpha>0$. Then there exists $T>0$ such that (1.2) has a solution $g(t)$ on $M \times[0, T)$ such that for each $t, g(t)$ has bounded curvature and is equivalent to $g_{0}$.

Proof. Apply the Proposition to find a short time solution $v$ of (1.1) with $\sigma(t)=-t \mathrm{Ric}_{0}+\omega_{0}$ and $f=0$. Then $\omega(t)=\sigma(t)+\sqrt{-1} \partial \bar{\partial} v$ is the required solution of the Kähler-Ricci flow.

\section{A PRIORI ESTIMATES}

Let $\left(M^{n}, g_{0}\right)$ be a smooth complete non-compact Kähler manifold with bounded geometry of order $2+\alpha$ for some $0<\alpha<1$. Let $f$ and $\sigma=\sigma(t)$ be a family of smooth functions and Kähler forms on $M \times[0, T)$ respectively. Let $f$ be a smooth function on $M \times[0, T)$. Let $v(x, t)$ be a smooth solution to the following equation on $M \times[0, T)$

$$
\left\{\begin{array}{l}
\frac{\partial v}{\partial t}=\log \frac{(\sigma+\sqrt{-1} \partial \bar{\partial} v)^{n}}{\omega_{0}^{n}}-f \text { in } M \times[0, T] \\
v(x, 0)=0
\end{array}\right.
$$

where $\omega_{0}$ is the Kähler form of $g_{0}$.

In this section, we want to obtain estimates on $v$. The derivations of the estimates are rather standard and along similar lines as in [22, 1] (compact case) [2, 6, 11] (non-compact case), except that we have a more simple proof for the second space derivatives of $v_{t}$ (see Lemma $3.4)$.

Throughout the section we will let $g_{k \bar{l}}(t)=(\sigma(t))_{k \bar{l}}+v_{k \bar{l}}$. We will use $\Delta, \nabla,|\cdot|$ and $\Delta_{\sigma}, \nabla_{\sigma},|\cdot|_{\sigma}$ to denote the Laplacian, covariant derivatives and norms with respect to $g$ and $\sigma$ respectively. 
Let us recall some well-known results, see [22, 2].

Lemma 3.1. Let $h(t)$ be a smooth family of uniformly equivalent complete Kähler metrics on $M$ for $t \in[0, T)$ and let $u$ be a smooth function on $M \times[0, T)$ such that $\widetilde{h}_{i \bar{\jmath}}=h_{i \bar{\jmath}}+u_{i \bar{\jmath}}$ is a family of complete Kähler metrics uniformly equivalent to $h$ for all $t$. In the following, $\widetilde{\nabla}, \widetilde{\Delta}$ and $\nabla_{h}, \Delta_{h}$ are covariant derivatives and Laplacians with respect to $\widetilde{h}$ and $h$ respectively. Also, $|\cdot|$ will denote a norm with respect to $h$.

(i)

$$
\widetilde{\Delta}\left(\Delta_{h} u+n\right) \geq \frac{\left|\widetilde{\nabla}\left(\Delta_{h} u+n\right)\right|^{2}}{\left(\Delta_{h} u+n\right)}-C\left(\Delta_{h} u+n\right) \tilde{h}^{i \bar{\jmath}} h_{i \bar{\jmath}}-h^{i \bar{\jmath}} \widetilde{R}_{i \bar{\jmath}}
$$

where $C$ is a constant depending only on $n$ and a bound on the holomorphic bisectional curvature of $h$ and $\widetilde{R}_{i \bar{\jmath}}$ is the Ricci curvature of $\tilde{h}$.

(ii) Let $Q=\widetilde{h}^{i \bar{j}} \widetilde{h}^{k l} \widetilde{h}^{m \bar{n}} u_{; i \bar{l} m} u_{; \bar{j} k \bar{n}}$, where ; is the covariant derivative with respect to $h$ and let

$$
F=\log \frac{\operatorname{det}\left(\widetilde{h}_{a \bar{b}}\right)}{\operatorname{det}\left(h_{a \bar{b}}\right)} .
$$

Then in normal coordinate with respect to $h$ :

$$
\begin{aligned}
\left(\widetilde{\Delta}-\frac{\partial}{\partial t}\right) Q \geq & \left(F_{; i \bar{k} m}-u_{t ; \bar{k} m}\right) u_{; \bar{i} k \bar{m}}+\left(F_{; \bar{i} k \bar{m}}-u_{t ; \bar{i} k \bar{m}}\right) u_{; i \bar{k} m} \\
& +\left(u_{t ; p \bar{k}}-F_{p \bar{k}}\right) u_{; i \bar{p} m} u_{; \bar{i} k \bar{m}}+\left(u_{t ; \bar{i} \bar{i}}-F_{p \bar{i}}\right) u_{; i \bar{k} m} u_{; \bar{p} k \bar{m}} \\
& +\left|u_{; i \bar{k} m \bar{a}}-u_{; \bar{k} p \bar{a}} u_{; \bar{i} m}\right|^{2} \\
& +\left|u_{; i \bar{k} m a}-\left(u_{; i \bar{p} a} u_{; p \bar{k} m}+u_{; m \bar{p} a} u_{; i \bar{k} p}\right)\right|^{2} \\
& \left.-C_{1}(n)\left[\left(\left|\nabla_{h} \operatorname{Rm}_{h}\right|+\left|\operatorname{Rm}_{h}\right|\right)\left|u_{i \bar{j}}\right|\left|u_{i \bar{j} k}\right|\right)+\left|\operatorname{Rm}_{h}\right|\left|u_{i \bar{j} k}\right|^{2}\right] \\
& -C_{2}\left|h_{t}\right|\left|u_{i \bar{j} k}\right|^{2}
\end{aligned}
$$

where $\mathrm{Rm}_{h}$ is the curvature tensor of $h$ and the last constant $C_{2}$ depends only on the equivalence of $\tilde{h}$ and $h$.

Lemma 3.2. Suppose $\Delta_{\sigma} v$ is bounded on $M \times\left[0, T^{\prime}\right]$ for all $T^{\prime}<T$ and suppose there exist $A_{i}$ such that in $M \times[0, T)$ :

$\left|v_{t}\right| \leq A_{1},|v| \leq A_{2},|f| \leq A_{3},\left|\Delta_{\sigma} f\right| \leq A_{4}, A_{5}^{-1} g_{0} \leq \sigma \leq A_{5} g_{0}, \quad\left|\sigma_{t}\right|_{\sigma} \leq A_{6}$, the holomorphic bisectional curvature of $\sigma$ is bounded by $A_{7}$, and the Ricci curvature $R_{i \bar{\jmath}}^{0}$ of $g_{0}$ is bounded by $A_{8}$. 
Then there is a positive constant $C$ depending only on $n$ and $A_{1}-A_{8}$ such that

$$
C^{-1} \leq n+\Delta_{\sigma} v \leq C
$$

on $M \times[0, T)$

Proof. Let $w=-v_{t}$. We have

$$
\begin{aligned}
n+\Delta_{\sigma} v & =\sigma^{i \bar{\jmath}}\left((\sigma)_{i \bar{\jmath}}+v_{i \bar{\jmath}}\right) \geq\left(\frac{\operatorname{det}\left(\sigma_{i \bar{\jmath}}+v_{i \bar{\jmath}}\right)}{\operatorname{det}\left(g_{0}\right)_{i \bar{\jmath}}}\right)^{\frac{1}{n}} \\
& \left.=\exp \left(\frac{1}{n}\left(f+v_{t}\right)\right)\right) \\
& \geq C_{0}
\end{aligned}
$$

where $C_{0}$ depends only on $n$ and $A_{1}$ and $A_{3}$. From this the first inequality in (3.4) is true. On the other hand, by Lemma 3.1 at a point with normal coordinate with respect to $\sigma(t)$ such that $g_{i \bar{\jmath}}(t)=(\sigma(t))_{i \bar{\jmath}}+v_{i \bar{\jmath}}=$ $\delta_{i j}\left(1+v_{i \bar{j}}\right)$ is diagonal, we have

$$
\begin{aligned}
\left(\Delta-\frac{\partial}{\partial t}\right) & {\left[\log \left(\Delta_{\sigma} v+n\right)\right] } \\
& \geq-C_{1} \sum_{i} \frac{1}{1+v_{i \bar{i}}}-\frac{\sigma^{i \bar{\jmath}} R_{i \bar{\jmath}}}{\Delta_{\sigma} v+n}-\frac{\frac{\partial}{\partial t} \Delta_{\sigma} v}{\Delta_{\sigma} v+n} \\
& =-C_{1} \sum_{i} \frac{1}{1+v_{i \bar{i}}}-\frac{\sigma^{i \bar{\jmath}} R_{i \bar{\jmath}}^{0}-\Delta_{\sigma} f-\left(\sigma_{t}^{i \bar{\jmath}}\right) v_{i \bar{\jmath}}}{\Delta_{\sigma} v+n} \\
& \geq-C_{1} \sum_{i} \frac{1}{1+v_{i \bar{i}}}-C_{2}
\end{aligned}
$$

Here $C_{1}$ is a constant depending only on $n$ and $A_{7}$ and $C_{2}$ is a constant depending only on $n, A_{1}, A_{3}, A_{4}, A_{5}, A_{7}, A_{8}$ where we have used (3.5), the fact that $u_{i \bar{i}}>-1$ for each $i$ and the fact that

$$
\Delta_{\sigma} v_{t}=-\sigma^{i \bar{\jmath}} R_{i \bar{\jmath}}+\sigma^{i \bar{\jmath}} R_{i \bar{\jmath}}^{0}-\Delta_{\sigma} f
$$


by (3.1). Hence for any $0 \leq t \leq T^{\prime}$

$$
\begin{aligned}
& \left(\Delta-\frac{\partial}{\partial t}\right)\left[\log \left(\Delta_{\sigma} v+n\right)-\left(C_{1}+1\right) v\right] \\
& \geq-C_{1} \sum_{i} \frac{1}{1+v_{i \bar{i}}}-C_{2}-\left(C_{1}+1\right) \sum_{i} \frac{v_{i \bar{i}}}{1+v_{i \bar{i}}}+\left(C_{1}+1\right) v_{t} \\
& =\sum_{i} \frac{1}{1+v_{i \bar{i}}}-\left(C_{1}+1\right) n-C_{2}+\left(C_{1}+1\right) v_{t} \\
& \geq\left(\frac{\sum_{i} 1+v_{i \bar{i}}}{\prod_{i}\left(1+v_{i \bar{i}}\right)}\right)^{\frac{1}{n-1}}-\left(C_{1}+1\right) n-C_{2}+\left(C_{1}+1\right) v_{t} \\
& \geq C_{3} \exp \left[\frac{1}{n-1}\left(\log \left(\Delta_{\sigma} v+n\right)-\left(C_{1}+1\right) v\right)\right]-C_{4} \\
& \geq C_{5}\left(\log \left(\Delta_{\sigma} u+n\right)-\left(C_{1}+1\right) v-C_{6}\right)
\end{aligned}
$$

where $C_{3}-C_{6}$ are positive constants depending only on $n$ and $A_{1}-A_{8}$, where we have used (3.5).

We now want to apply a maximum principle argument to (3.7). The argument is basically similar to that in [15] where maximum principles were derived for the case where $g(t)$ is evolving by Kähler-Ricci flow, except that in our case we do not assume the curvature of $g$ is bounded. Since $g_{0}$ has bounded curvature, we can find a function $\phi$ as in Lemma (ii). At a point we can find holomorphic coordinates such that $g_{i \bar{\jmath}}$ is diagonalized at this point with respect to $g_{0}$. Combining (3.5) with our assumption that $\Delta_{\sigma} v$ is a bounded function for each $t<T$, it follows that $g(t)$ and $g_{0}$ are uniformly equivalent for each $t<T$. Then $\Delta \phi=g^{i \bar{j}} \phi_{i j}=g^{i \bar{i}} \phi_{i \bar{i}}$ is bounded in $M \times\left[0, T^{\prime}\right]$ for all $T^{\prime}<T$. Hence $\Delta \phi \leq \alpha \phi$, where $\alpha$ may depending on $T$. Consider $h=e^{\alpha t} \phi$ we have

$$
\left(\Delta-\frac{\partial}{\partial t}\right) h \leq 0 .
$$

Consider the function

$$
H=\left(\log \left(\Delta_{\sigma} u+n\right)-\left(C_{1}+1\right)-C_{6}\right)-\epsilon h
$$

for $\epsilon>0$ Then

$$
\left(\Delta-\frac{\partial}{\partial t}\right) H \geq C_{5}\left(\log \left(\Delta_{\sigma} v+n\right)-\left(C_{1}+1\right) v+-C_{6}\right)
$$

for $T \geq t>0$. On $M \times[0, T], H$ is bounded from above and will tend to $-\infty$ at infinity by $(3.5)$ and the fact that $v$ is bounded. $H$ must attains its maximum. Hence by the maximum principle, $H$ cannot 
attain positive maximum at $t>0$. Hence

$$
\sup _{M \times[0, T]} H \leq \max \left\{\log n-C_{6}, 0\right\} .
$$

The second inequality in (3.7) is true by letting $\epsilon \rightarrow 0$.

Corollary 3.1. Assume the hypothesis and notation in Lemma 3.2. Then here exists a positive constants $C>0$ depending on $n$ and $A_{1}-A_{8}$ in Lemma 3.2 such that

$$
C^{-1} g_{0} \leq g \leq C g_{0}, \text { and }\left|v_{i \bar{\jmath}}\right|_{\sigma} \leq C
$$

on $M \times[0, T)$.

Lemma 3.3. Assume the hypothesis and notation in Lemma 3.2. In addition assume there exist $A_{9}$ and $A_{10}$ such that on $M \times[0, T)$,

$$
\left|\nabla_{\sigma}^{2} f\right|_{\sigma}+\left|\nabla_{\sigma}^{3} f\right|_{\sigma} \leq A_{9},\left|\mathrm{Rm}^{\sigma}\right|+\left|\nabla_{\sigma} \mathrm{Rm}^{\sigma}\right| \leq A_{10},
$$

where $\mathrm{Rm}^{\sigma}$ is the curvature tensor of $\sigma$. Suppose that $\left|v_{; i \bar{k} k}\right|_{\sigma}$ is bounded on $M \times\left[0, T^{\prime}\right]$ for all $T^{\prime}<T$.

Then there is a constant $C$ depending only $n$ and $A_{1}-A_{10}$ such that

$$
Q=g^{i \bar{j}} g^{k \bar{l}} g^{m \bar{n}} v_{; i \bar{l} m} v_{; \bar{j} k \bar{n}} \leq C
$$

on $M \times[0, T)$.

Proof. In the following $C_{i}$ 's denote positive constants depending only on $n$ and $A_{1}-A_{10}$. For $0 \leq t<T$, by Lemma 3.1 we have that in normal coordinates with respect to $\sigma$,

$$
\begin{aligned}
(\Delta- & \left.\frac{\partial}{\partial t}\right) Q \\
\geq & \left(F_{; i \bar{k} m}-v_{t ; i \bar{k} m}\right) v_{; \bar{i} k \bar{m}}+\left(F_{; \bar{i} k \bar{m}}-v_{t ; \bar{i} k \bar{m}}\right) v_{; i \bar{k} m} \\
& +\left(v_{t ; p \bar{k}}-F_{p \bar{k}}\right) v_{; i \bar{p} m} v_{; \bar{i} k \bar{m}}+\left(v_{t ; \bar{i}}-F_{p \bar{i}}\right) v_{; i \bar{k} m} v_{; \bar{p} k \bar{m}} \\
& +\left|v_{; i \bar{k} m \bar{a}}-v_{; \bar{k} p \bar{a}} v_{; i \bar{p} m}\right|_{\sigma}^{2} \\
& +\left|v_{; i \bar{k} m a}-\left(v_{; i \bar{p} a} v_{; p \bar{k} m}+v_{; m \bar{p} a} v_{; i \bar{k} p}\right)\right|_{\sigma}^{2} \\
& \left.-C(n)\left[\left(\left|\nabla_{\sigma} \operatorname{Rm}^{\sigma}\right|_{\sigma}+\left|\mathrm{Rm}^{\sigma}\right|_{\sigma}\right)\left|v_{i \bar{j}}\right|_{\sigma}\left|v_{i \bar{j} k}\right|_{\sigma}\right)+\left|\mathrm{Rm}^{\sigma}\right|\left|v_{i \bar{j} k}\right|_{\sigma}^{2}\right] \\
& +C_{0}\left|v_{i \bar{j} k}\right|_{\sigma}^{2} \\
\geq & -C_{1} Q-C_{2}+\left|v_{; i \bar{k} m \bar{a}}-v_{; \bar{k} p \bar{a}} v_{; i \bar{p} m}\right|_{\sigma}^{2} \\
& +\left|v_{; i \bar{k} m a}-\left(v_{; i \bar{p} a} v_{; p \bar{k} m}+v_{; m \bar{p} a} v_{; i \bar{k} p}\right)\right|_{\sigma}^{2} .
\end{aligned}
$$


On the other hand, direct computations show:

$$
\begin{aligned}
(\Delta & \left.-\frac{\partial}{\partial t}\right)\left(\Delta_{\sigma} v+n\right) \\
& =g^{k \bar{l}} g^{p \bar{q}} v_{; p \bar{i} l} v_{i \bar{q} \bar{k}}-g^{k \bar{l}} g_{i \bar{\jmath}} R_{i \bar{j} k \bar{l}}^{\sigma}-\sigma^{i \bar{\jmath}} R_{i \bar{\jmath}}-\Delta_{\sigma} v_{t}-\left(\sigma^{i \bar{\jmath}}\right)_{t} v_{i \bar{\jmath}} \\
& =g^{k \bar{l}} g^{p \bar{q}} v_{; p \bar{i} l} v_{i \bar{q} \bar{k}}-g^{k \bar{l}} g_{i \bar{\jmath}} R_{i \bar{j} k \bar{l}}^{\sigma}-\sigma^{i \bar{\jmath}} R_{i \bar{\jmath}}^{0}+\Delta_{\sigma} f-\left(\sigma^{i \bar{\jmath}}\right)_{t} v_{i \bar{\jmath}} \\
& \geq C_{3} Q-C_{4}
\end{aligned}
$$

where we have used (3.1) and Corollary 3.1. Hence by Lemma 3.2

$$
\left(\Delta-\frac{\partial}{\partial t}\right)\left(Q+\frac{C_{1}+1}{C_{3}}\left(\Delta_{\sigma} v+n\right)\right) \geq Q+\frac{C_{1}+1}{C_{3}}\left(\Delta_{\sigma} v+n\right)-C_{5} .
$$

By assumption and Corollary 3.1, $Q+\frac{C_{1}+1}{C_{3}}\left(\Delta_{\sigma} u+n\right)$ is bounded on $M \times\left[0, T^{\prime}\right]$ for all $T^{\prime}<T$. As in the proof of Lemma 3.2, we can conclude that the lemma is true by the maximum principle.

Now we want to estimate the function $w=-v_{t}$. We begin by noting the following estimate which follows immediately from Lemma 3.3, (3.1) and (3.8):

Corollary 3.2. With the same assumptions and notations as in Lemma 3.3. In addition, suppose there exist $A_{11}$ and $A_{12}$ such that on $M \times[0, T)$

$$
\left|\nabla_{0} \sigma\right|_{\sigma} \leq A_{11},\left|\nabla_{\sigma} f\right|_{\sigma} \leq A_{12}
$$

where $\nabla_{0}$ is the covariant derivative with respect to $g_{0}$. Then there exists a positive constants $C>0$ depending on $n$ and and $A_{1}-A_{12}$ such that $|\nabla w|^{2} \leq C$ on $M \times[0, T)$.

Now we want to estimate higher order derivatives ow $w$. By (3.1) we note that

$$
\left(\Delta-\frac{\partial}{\partial t}\right) w=g^{i \bar{\jmath}}\left(\sigma_{t}\right)_{i \bar{\jmath}}-f_{t}:=F
$$

and

$$
w_{i \bar{\jmath}}=R_{i \bar{\jmath}}-R_{i \bar{\jmath}}^{0}+f_{i \bar{\jmath}}=R_{i \bar{\jmath}}-\Omega_{i \bar{\jmath}},
$$

where $\Omega_{i \bar{\jmath}}=R_{i \bar{\jmath}}^{0}-f_{i \bar{\jmath}}$. Now we compute $\left(\Delta-\frac{\partial}{\partial t}\right)|\nabla w|^{2}$. In normal coordinates with respect to $g$ we have 


$$
\begin{aligned}
\Delta\left(|\nabla w|^{2}\right) & =g^{i \bar{\jmath}}\left(w_{k} w_{\bar{l}} g^{k \bar{l}}\right)_{i \bar{\jmath}} \\
& =g^{i \bar{\jmath}} g^{k \bar{l}}\left(w_{k i} w_{\overline{l j}}+w_{k \bar{j}} w_{\bar{l}}+w_{k i \bar{j}} w_{\bar{l}}+w_{k} w_{\bar{l} \bar{\jmath}}\right)+g^{i \bar{\jmath}} w_{k} w_{\bar{l}}\left(g^{k \bar{l}}\right)_{i \bar{\jmath}} \\
& =\sum_{i, j}\left(\left|w_{i j}\right|^{2}+\left|w_{i \bar{\jmath}}\right|^{2}\right)+(\Delta)_{i} w_{\bar{i}}+(\Delta w)_{\bar{i}} w_{i}+R_{i \bar{\jmath}} w_{i} w_{\bar{j}} \\
& =\sum_{i, j}\left(\left|w_{i j}\right|^{2}+\left|w_{i \bar{\jmath}}\right|^{2}\right)+(\Delta)_{i} w_{\bar{i}}+(\Delta w)_{\bar{i}} w_{i}+w_{i \bar{\jmath}} w_{i} w_{\bar{j}}+\Omega_{i \bar{\jmath}} w_{i} w_{\bar{j}}
\end{aligned}
$$

where we have used (3.14).

$$
\begin{aligned}
\frac{\partial}{\partial t}\left(|\nabla w|^{2}\right) & =g^{i \bar{\jmath}}\left(w_{i t} w_{\bar{j}}+w_{i} w_{\bar{j} t}\right)+\left(g^{i \bar{\jmath}}\right)_{t} w_{i} w_{\bar{j}} \\
& =\left(w_{t}\right)_{i} w_{\bar{i}}+\left(w_{t}\right)_{\bar{i}} w_{i}+\left(w_{i \bar{\jmath}}-\sigma_{i \bar{\jmath}}\right) w_{i} w_{\bar{j}}
\end{aligned}
$$

Hence

$$
\begin{aligned}
\left(\Delta-\frac{\partial}{\partial t}\right)\left(|\nabla w|^{2}\right)= & \sum_{i, j}\left(\left|w_{i j}\right|^{2}+\left|w_{i \bar{\jmath}}\right|^{2}\right)+\left(\Omega_{i \bar{\jmath}}+\sigma_{i \bar{\jmath}} w_{i} w_{\bar{j}}\right) \\
& +F_{i} w_{\bar{i}}+F_{\bar{i}} w_{i} .
\end{aligned}
$$

Lemma 3.4. Assume the hypothesis and notation in Corollary 3.2. Assume in addition that there exist $A_{13}$ and $A_{14}$ such that on $M \times[0, T)$,

$$
\left|\nabla_{0} \sigma_{t}\right|_{\sigma}+\left|\nabla_{0}^{2} \sigma_{t}\right|_{\sigma} \leq A_{13},\left|\left(f_{t}\right)_{i \bar{\jmath}}\right|_{\sigma} \leq A_{14} .
$$

Suppose that $S:=g^{i \bar{l}} g^{k \bar{j}} w_{i \bar{\jmath}} w_{k \bar{l}}$ is bounded on $M \times\left[0, T^{\prime}\right]$ for all $T^{\prime}<T$.

Then there is a constant $C$ depending only on $n$ and $A_{1}-A_{14}$, such that $S \leq C$ on $M \times[0, T)$.

Proof. In the following $C_{i}$ will denote positive constants depending only on $n$ and $A_{1}-A$. By (3.13) and Lemma 2.1 in 12 ]

$$
\begin{aligned}
\left(\Delta-\frac{\partial}{\partial t}\right) w_{i \bar{\jmath}} & =R_{l \bar{k} i \bar{j}} w_{k \bar{l}}-\frac{1}{2}\left(R_{i \bar{p}} w_{p \bar{j}}+R_{p \bar{j}} w_{i \bar{l}}\right)+F_{i \bar{\jmath}} \\
& =R_{l \bar{k} i \bar{j}} w_{k \bar{l}}-\frac{1}{2}\left(w_{i \bar{p}} w_{p \bar{j}}+w_{p \bar{j}} w_{i \bar{l}}\right)-\frac{1}{2}\left(\Omega_{i \bar{p}} w_{p \bar{j}}+\Omega_{p \bar{j}} w_{i \bar{l}}\right)+F_{i \bar{\jmath}}
\end{aligned}
$$


where we have used (3.14). In normal coordinates with respect to $g$ :

$$
\begin{aligned}
(\Delta- & \left.\frac{\partial}{\partial t}\right) S \\
= & w_{i \bar{j} k} w_{\bar{i} j \bar{k}}+w_{\bar{j} i \bar{k}} w_{j \bar{i} k}+w_{j \bar{i}} \Delta w_{i \bar{j}}+w_{i \bar{j}} \Delta w_{j \bar{i}} \\
& \quad-w_{j \bar{i}} \frac{\partial}{\partial t} w_{i \bar{\jmath}}-w_{i \bar{j}} \frac{\partial}{\partial t} w_{j \bar{i}}-g^{i \bar{r}} g^{s \bar{l}} g^{k \bar{j}} w_{s \bar{r}} w_{i \bar{\jmath}} w_{k \bar{l}}-g^{i \bar{l}} g^{k \bar{r}} g^{s \bar{\jmath}} w_{s \bar{r}} w_{i \bar{\jmath}} w_{k \bar{l}} \\
= & 2\left|w_{i \bar{j} k}\right|^{2}+2 w_{i \bar{\jmath}}\left(R_{l \bar{k} i \bar{\jmath}} w_{k \bar{l}}-\frac{1}{2}\left(w_{i \bar{p}} w_{p \bar{j}}+w_{p \bar{j}} w_{i \bar{l}}\right)\right) \\
& \quad-w_{i \bar{\jmath}}\left(\Omega_{i \bar{p}} w_{p \bar{j}}+\Omega_{p \bar{j}} w_{i \bar{l}}+2 F_{i \bar{\jmath}}\right)-g^{i \bar{r}} g^{s \bar{l}} g^{k \bar{j}} w_{s \bar{r}} w_{i \bar{j}} w_{k \bar{l}}-g^{i \bar{l}} g^{k \bar{r}} g^{s \bar{\jmath}} w_{s \bar{r}} w_{i \bar{\jmath}} w_{k \bar{l}} .
\end{aligned}
$$

Hence

$$
\begin{aligned}
(\Delta & \left.-\frac{\partial}{\partial t}\right)(1+S)^{\frac{1}{2}} \\
= & \frac{1}{2(1+S)^{\frac{1}{2}}}\left(\Delta-\frac{\partial}{\partial t}\right) S-\frac{|\nabla S|^{2}}{(1+S)^{\frac{3}{2}}} \\
\geq & \frac{1}{2(1+S)^{\frac{1}{2}}}\left[2 w_{i \bar{\jmath}}\left(R_{l \bar{k} i \bar{\jmath}} w_{k \bar{l}}-\frac{1}{2}\left(w_{i \bar{p}} w_{p \bar{j}}+k w_{p \bar{j}} w_{i \bar{l}}\right)\right)\right. \\
& \left.-w_{i \bar{\jmath}}\left(\Omega_{i \bar{p}} w_{p \bar{j}}+\Omega_{p \bar{j}} w_{i \bar{l}}+2 F_{i \bar{\jmath}}+w_{i \bar{\jmath}}\right)\right] \\
& -\frac{1}{2(1+S)^{\frac{1}{2}}}\left[g^{i \bar{r}} g^{s \bar{l}} g^{k \bar{j}} w_{s \bar{r}} w_{i \bar{\jmath}} w_{k \bar{l}}-g^{i \bar{l}} g^{k \bar{r}} g^{s \bar{\jmath}} w_{s \bar{r}} w_{i \bar{\jmath}} w_{k \bar{l}}\right] \\
\geq & -C_{1}\left(S+|\mathrm{Rm}|^{2}+\left|F_{i \bar{\jmath}}\right|^{2}\right) .
\end{aligned}
$$

for some constant $C_{1}$ depending only on $n$ and $A_{1}-A$, where we have used the fact that $|\nabla S|^{2} \leq 2 S \sum_{i, j, k}\left|w_{i \bar{j} k}\right|^{2}$.

In order to estimate $\left|F_{i j}\right|$, note that in normal coordinate of $\sigma$ at a point,

$$
R_{i \bar{j} k \bar{l}}=g^{p \bar{q}} \frac{\partial g_{p \bar{j}}}{\partial z_{l}} \frac{\partial g_{i \bar{q}}}{\partial \bar{z}_{k}}+R_{i \bar{j} k \bar{l}}^{\sigma}-\frac{\partial^{2}}{\partial z_{k} \partial \bar{z}_{l}} v_{i \bar{\jmath}} .
$$

Using Corollary 3.1, Lemma 3.3 we have

$$
\left|\frac{\partial^{2}}{\partial z_{k} \partial \bar{z}_{l}} v_{i \bar{\jmath}}\right| \leq C_{2}(|\mathrm{Rm}|+1)
$$

for some constant $C_{2}$ depending only on the constants in the lemma. Now in a normal coordinate of $\sigma$ we have

$$
\begin{aligned}
F_{i \bar{\jmath}} & =\left(g^{k \bar{l}}\left(\sigma_{t}\right)_{k \bar{l}}\right)_{i \bar{\jmath}}+\left(f_{t}\right)_{i \bar{\jmath}} \\
& =\left(g^{k \bar{l}}\right)_{i \bar{\jmath}}\left(\sigma_{t}\right)_{k \bar{l}}+\left(g^{k \bar{l}}\right)_{i}\left(\left(\sigma_{t}\right)_{k \bar{l}}\right)_{\bar{j}}+\left(g^{k \bar{l}}\right)_{\bar{j}}\left(\left(\sigma_{t}\right)_{k \bar{l}}\right)_{i}+\left(g^{k \bar{l}}\right)\left(\left(\sigma_{t}\right)_{k \bar{l}}\right)_{i \bar{\jmath}}+\left(f_{t}\right)_{i \bar{\jmath}}
\end{aligned}
$$


Hence by (3.22), we have

$$
\left|F_{i \bar{j}}\right| \leq C_{3}(|\mathrm{Rm}|+1)
$$

On the other hand, by Corollary 3.1, Lemma 3.3, (3.11), (3.21) and (3.3) we have:

$$
\left(\Delta-\frac{\partial}{\partial t}\right) Q \geq C(n)|\mathrm{Rm}|^{2}-C_{4}
$$

By (3.17), (3.20), (3.24), (3.23), Lemma 3.3, Corollary 3.1 and Corollary $\left[3.2\right.$, we can find positive constants $C_{5}, C_{6}, C_{7}$ depending only on the quantities in the lemma such that in $M \times[0, T]$

$$
\begin{aligned}
\left(\Delta-\frac{\partial}{\partial t}\right) & {\left[(1+S)^{\frac{1}{2}}+C_{5}|\nabla w|^{2}+C_{6} Q\right] } \\
& \geq\left[(1+S)^{\frac{1}{2}}+C_{5}|\nabla w|^{2}+C_{6} Q\right]-C_{7}
\end{aligned}
$$

where we have used (3.17). By assumption, for each $t \in[0, T), S$ is bounded. One may then proceed as in the proof of Lemma 3.2 to conclude that

$$
S \leq C
$$

for some constant $C$ depending only on the constants mentioned in the lemma.

From the above Lemmas we may conclude

Corollary 3.3. Let $v$ be as in Lemma 3.4. There is a constant $C$ depending only on the quantities in Lemma 3.4 such that

$$
\|v\|_{2+\alpha, 1+\alpha / 2, M \times[0, T)} \leq C
$$

Remark 2. Let $\Omega$ be a bounded domain. Suppose the quantities that we want to estimates in this section are bounded in $\partial \Omega \times[0, T)$. Then it is easy to see that from the above proofs we may conclude that the quantities are also bounded in $\Omega \times[0, T)$.

\section{Applichtions}

We will now apply the results of the previous sections. Given a solution $v(x, t)$ to (3.1) as in the previous section, we are interested in establishing conditions under which we have longtime existence of $v(x, t)$ as a solution to (3.1). More generally we are also interested in where singularities can form when (3.1) does not admit a longtime solution. In Theorem 4.1 below we describe where singularities of (3.1) can occur in the case where our solution corresponds to a solution to 
the Kähler-Ricci flow. As a corollary we will establish a longtime existence result for the Kähler-Ricci flow in Corollary 4.1 which improves the longtime existence result in [5]. Then in Theorem 4.2, combining the $C^{0}$ estimate in [6] with the a priori estimates of the previous section we establish a longtime convergent solution to (3.1) under certain conditions which generalize those in the main result in [6].

The following Theorem describes where singularities can or cannot form under the Kähler-Ricci flow in terms of the existence of plurisubharmonic functions defined on subsets of a complete non-compact Kähler manifold.

Theorem 4.1. Let $\left(M, g_{0}\right)$ be a complete non-compat Kähler manifold such that

i) $\left|R m_{0}(x)\right| \rightarrow 0$ as $d(x) \rightarrow \infty$ where $d(x)$ is the distance function on $M$ from some $p \in M$.

ii) The injectivity radius of $\left(M, g_{0}\right)$ is uniformly bounded below by some $c>0$.

iii) There exists an open set $S$ with smooth compact boundary $\partial S$ and a smooth function $F$ which is strictly pluri-subharmonic on $S$ and smooth up to $\partial S$.

Let $g(t)$ be a solution to the Kähler-Ricci flow $g^{\prime}=-R c$ on $M \times[0, T)$ with initial condition $g(0)=g_{0}$. Then for any closed set $N$ contained in $S$, the Riemannian curvature tensor of $g(t)$ and all its covariant derivatives are bounded on $N \times[0, T)$ provided they are bounded on $\partial S \times[0, T)$.

Proof. By the results in [15, we may assume that $g_{0}$ is smooth and that all the covariant derivatives of the curvature tensor of $\omega_{0}$ are bounded on $M$. Now suppose $g(t)$ solves the Kähler-Ricci flow $g^{\prime}=-R c$ on $M \times[0, T)$ with initial condition $g(0)=g_{0}$, and that the curvature of $g(t)$ all its covariant derivatives are bounded on $\partial S \times[0, T)$.

By the discussion in the introduction, we know that there is a solution $v(x, t)$ to

$$
\left\{\begin{array}{l}
\frac{\partial v}{\partial t}=\log \frac{(\sigma+\sqrt{-1} \partial \bar{\partial} v)^{n}}{\left(\omega_{0}\right)^{n}} \\
v(x, 0)=0
\end{array}\right.
$$

on $M \times[0, T)$ such that $\sigma=-t R c_{0}+\omega_{0}$ and $\omega=\sigma+\sqrt{-1} \partial \bar{\partial} v$ where $\omega$ is the Kähler form for $g(t)$. By Theorem 9.1 in [5] we can find sufficiently large bounded open sets $\Omega_{1}, \Omega$ with smooth boundary such that

(i) $\partial S \subset \Omega_{1} \subset \subset \Omega$; 
(ii) $\left|\nabla_{t}^{k} R m(x, t)\right| \leq C_{k}$ on $\Omega_{1}^{c} \times[0, T)$ for all $k$ and some $C_{k}$ depending only on $\Omega$ and $k$.

In particular, from our hypothesis and the definition of $\sigma$ it is not hard to show we thus have $\left|\nabla_{0}^{k} v\right| \leq C_{k}$ on $\partial(\Omega \bigcap S) \times[0, T)$ for all $k$ and some $C_{k}$ depending only on $k$ and $T$. Now if $N$ is any closed subset of $S$, then we have $N=\left(\Omega_{1} \bigcap N\right) \bigcup\left(\Omega_{1}^{c} \bigcap N\right)$ where $\Omega_{1} \bigcap N$ has compact closure in $\Omega \bigcap S$. Hence by condition (ii) above, we see that to prove the theorem it will be sufficient to prove that $\left|\nabla_{0}^{k} v\right| \leq C_{k}$ on $\Omega^{\prime} \times[0, T)$ for all $k$ and some $C_{k}$ depending only on $g_{0}, k, T$ and $\Omega^{\prime}$ where $\Omega^{\prime}$ is any closed subset of $\Omega \bigcap S$. We now proceed to do this. We begin by showing

Claim 1: There exists $C>0$ such that $\sup _{(\Omega \cap S) \times[0, T)}\left|v_{t}\right| \leq C$.

We will establish the claim by using the a priori estimates from the previous section applied to domains (see Remark 2), and by using the plurisubharmonic function $F$ and arguing as in [11] where the authors considered the normalized Kähler-Ricci flow $g^{\prime}=-R c-g$ on a complete non-compact Kähler manifold (also see [20] and [21]).

We begin by differentiating (4.1) with respect to $t$ successively to obtain the following which we express in an orthonormal coordinate with respect to $g(t)$ :

$$
\begin{gathered}
\frac{\partial v_{t}}{\partial t}=\Delta v_{t}+\left(\sigma^{\prime}\right)_{i \bar{i}} \\
\frac{\partial v_{t t}}{\partial t}=\Delta v_{t t}-\left(g^{\prime}(t)\right)_{i \bar{j}}\left(v_{t}\right)_{i \bar{j}}-\left(g^{\prime}(t)\right)_{i \bar{j}}\left(\sigma^{\prime}\right)_{i \bar{j}} \\
=\Delta v_{t t}-\left(g^{\prime}(t)\right)_{i \bar{j}}\left(g^{\prime}(t)\right)_{i \bar{j}} \\
\leq \Delta v_{t t}
\end{gathered}
$$

Now from our above observation and (4.1), we know that $\left|v_{t t}\right|$ is bounded on $\partial(\Omega \bigcap S) \times[0, T)$. From this fact and (4.3), we conclude by the maximum principle that $v_{t t}$, and thus $v_{t}$ and $v$ is bounded from above on $(\Omega \bigcap S) \times[0, T)$. Now for the bound from below, we use the plurisubharmonic function $F$ and compute in an orthonormal coordinate with respect to $g(t)$ : 


$$
\begin{aligned}
\frac{\partial}{\partial t}\left((2 T-t) v_{t}+v-F\right)= & \Delta\left((2 T-t) v_{t}+v-F\right) \\
& -\Delta u+\Delta F+2 T\left(\sigma_{t}^{\prime}\right)_{i \bar{i}}-t\left(\sigma_{t}^{\prime}\right)_{i \bar{i}} \\
= & \Delta\left((2 T-t) v_{t}+v-F\right)-n+(2 T \sigma)_{i \bar{i}}+F_{i \bar{i}} \\
\geq & \Delta\left((2 T-t) v_{t}+v-F\right)-n
\end{aligned}
$$

where in the second equality we have used the fact that by scaling $F$ we may assume that we have $2 T\left(\sigma_{t}^{\prime}\right)_{i \bar{i}}+F_{i \bar{i}} \geq 0$ on $(\Omega \bigcap S), \sigma^{\prime}=-R c_{0}$ and the fact that $n=g^{i \bar{j}} g_{i \bar{j}}=g^{i \bar{j}}(\sigma+\partial \bar{\partial} v)_{i \bar{j}}=\Delta v-t g^{i \bar{j}}\left(R c_{0}\right)_{i \bar{j}}+g^{i \bar{j}}\left(\omega_{0}\right)_{i \bar{j}}$. Arguing as above and using the bound on $v$ from above, we conclude from (4.4) and the maximum principle that $v_{t}$ and thus $v$ is bounded from below on $(\Omega \bigcap S) \times[0, T)$. This completes the proof of the claim.

We now modify (4.1) so that the varying background metric is uniformly Kähler. We note that

$$
\sigma=\frac{T-t}{T} \sigma(0)+\frac{t}{T} \sigma(T)
$$

and we let

$$
\begin{aligned}
\hat{\sigma} & =\frac{T-t}{T} \sigma(0)+\frac{t}{T}(\sigma(T)+\partial \bar{\partial} F) \\
\hat{v} & =v-\frac{t}{T} F .
\end{aligned}
$$

By condition (iii), by scaling $F$ we may assume that $\sigma(T)+\partial \bar{\partial} F$ is equivalent to $\omega_{0}$ on $(\Omega \bigcap S)$. Hence $\hat{\sigma}$ is uniformly equivalent to $\omega_{0}$ on $(\Omega \bigcap S) \times[0, T)$.

Then from the above equations and (4.1) we have

$$
\left\{\begin{array}{l}
\frac{\partial \hat{v}}{\partial t}=\log \frac{(\hat{\sigma}+\partial \bar{\partial} \hat{v})^{n}}{\left(\omega_{0}\right)^{n}}-\frac{F}{T} \\
\hat{v}(x, 0)=0 .
\end{array}\right.
$$

The point is that the background $\hat{\sigma}$ is now a Kähler metric which is uniformly equivalent to $\omega_{0}$ on $(\Omega \bigcap S) \times[0, T)$. On the other hand, our previous estimates imply that $\left|\hat{v}_{t}\right|$ and thus $|\hat{v}|$ are uniformly bounded on $(\Omega \bigcap S) \times[0, T)$. From this fact, the above observation that $\left|\nabla_{0}^{k} v\right|$ and thus $\left|\nabla_{0}^{k} \hat{v}\right|$ is uniformly bounded on $\partial(\Omega \bigcap S) \times[0, T)$ for all $k$, and the estimates in the previous section we can conclude by the maximum principle that $\hat{v}_{i \bar{\jmath}}, \hat{v}_{i \bar{\jmath} k}, \hat{v}_{t i \bar{\jmath}}$ are uniformly bounded on $(\Omega \bigcap S) \times[0, T)$. Thus by differentiating (4.7) and applying parabolic Schauder estimates we conclude that $\left|\nabla_{0}^{k} \hat{v}\right|$ and thus $\left|\nabla_{0}^{k} v\right|$ is uniformly bounded on $\Omega^{\prime} \times[0, T)$ for all $k=0,1,2, .$. by a constant depending only on $T$, 
$g_{0}, \Omega^{\prime}$ and $k$ where $\Omega^{\prime}$ is any closed set in $(\Omega \bigcap S)$. This completes the proof of the Theorem.

As a Corollary of Theorem 4.1 we have the following longtime existence result for the Kähler-Ricci flow.

Corollary 4.1. Let $\left(M, g_{0}\right)$ be a complete non-compat Kähler manifold such that

i) $\left|R m_{0}(x)\right| \rightarrow 0$ as $d(x) \rightarrow \infty$ where $d(x)$ is the distance function on $M$ from some $p \in M$.

ii) The injectivity radius of $\left(M, g_{0}\right)$ is uniformly bounded below by some $c>0$.

iii) There exists a strictly pluri-subharmonic function $F$ on $M$.

Then the Kähler-Ricci flow $g^{\prime}=-$ Rc has a longtime solution $g(t)$ on $M$ with initial condition $g(0)=g_{0}$.

We now turn to our second application of the a priori estimates from our previous section. We will establish the following convergence result for (3.1) which generalizes the main convergence result in [6].

Theorem 4.2. Let $\left(M^{n}, g_{0}\right)$ be a smooth complete non-compact Kähler manifold with $n \geq 3$, and let $\Omega$ be a (1,1)-form on $M$ such that Ric $_{0}-\Omega=\sqrt{-1} \partial \partial \bar{\partial} f_{0}$ for some smooth potential $f_{0}$. Suppose we have $\sum_{k=0}^{1}\left|\nabla_{0}^{k} R m\right|+\sum_{k=0}^{3}\left|\nabla_{0}^{k} f\right|<\infty$. Then we have the following:

1. The following modified Kähler-Ricci flow has a long time smooth solution $g(t)$.

$$
\left\{\begin{array}{l}
\frac{\partial g_{i \bar{\jmath}}}{\partial t}=-R_{i \bar{\jmath}}+\Omega_{i \bar{\jmath}} \\
g_{i \bar{\jmath}}(x, 0)=\left(g_{0}\right)_{i \bar{\jmath}}
\end{array}\right.
$$

2. If in addition the potential $f_{0}$ satisfies

(a)

$$
\left|f_{0}\right|(x) \leq \frac{C_{1}}{1+\rho_{0}^{2+\epsilon}(x)}
$$

for some $C_{1}, \epsilon>0$, and all $x \in M$ where $\rho_{0}(x)$ is the distance function from a fixed $o \in M$.

(b) The following Sobolev inequality is true:

$$
\left(\int_{M}|\phi|^{\frac{2 n}{n-1}} d V_{0}\right)^{\frac{n-1}{n}} \leq C_{2} \int_{M}\left|\nabla_{0} \phi\right|^{2} d V_{0}
$$

for some $C_{2}>0$ and all $\phi \in C_{0}^{\infty}(M)$. 
(c) There exists a constant $C_{3}>0$ such that

$$
V_{0}(r) \leq C_{3} r^{2 n}
$$

for some $C_{3}>0$ and all $r$ where $V_{0}(r)$ is the volume of the geodesic ball with radius $r$ centered at some $o \in M$.

Then as $t \rightarrow \infty, g(t)$ converges uniformly on compact sets in the $C^{\infty}$ topology on $M$ to a complete Kähler-Ricci metric $g_{\infty}$ on $M$ which is uniformly equivalent to $g_{0}$, has bounded geometry of order $2+\alpha$ and has Ricci form equal to $\Omega$.

Remark 3. If we assume further that all the covariant derivatives of $R m_{0}$ and $f_{0}$ are bounded, then $g_{\infty}$ will also have all covariant derivatives of curvature bounded.

As in [6] our approach is to consider (3.1) where we set $\sigma=g_{0}$. In other words, we consider the equation

$$
\left\{\begin{array}{l}
\frac{\partial v}{\partial t}=\log \frac{\operatorname{det}\left(\left(g_{0}\right)_{k \bar{l}}+v_{k \bar{l}}\right)}{\operatorname{det}\left(\left(g_{0}\right)_{k \bar{l}}\right)}-f_{0} \\
v(x, 0)=0
\end{array}\right.
$$

A straight forward calculation show that if $v(x, t)$ solves (4.12) on $M \times$ $[0, T)$ then $g_{i \bar{\jmath}}(t)=\left(g_{0}\right)_{i \bar{\jmath}}+v_{i \bar{\jmath}}$ is a family of Kähler metrics on $M$ which solves (4.8) on $M \times[0, T)$. On the other hand, it is not hard to show that given a solution $g_{i j}(t)$ to (4.8) on $M \times[0, T)$, then we obtain a solution $v(x, t)$ to (4.12) on $M \times[0, T)$ (see [6]).

We now prove the first part of the Theorem. First note that the assumptions on the curvature tensor imply $\left(M, g_{0}\right)$ has bounded geometry of order $2+\alpha$ for some $0<\alpha<1$ by [19]. The assumptions on $f$ then imply that $f \in C^{2+\alpha}(M)$. Hence under the hypothesis in the theorem, by Proposition 2.1 there exists a maximal smooth solution $v$ to (4.12) satisfying the conclusions of the Proposition on $M \times[0, T)$ for some $T>0$. On the other hand, differentiating (4.12) with respect to $t$ gives

$$
\left\{\begin{array}{c}
\frac{\partial v_{t}}{\partial t}=\Delta_{t} v_{t} \\
v_{t}(x, 0)=f_{0}
\end{array}\right.
$$

and thus by the maximum principle and our hypothesis on $f_{0}$, we conclude that $\left|v_{t}\right|$ and thus $|v|$ is uniformly bounded on $M \times[0, T)$. Now by Remark 1, we may have that the conclusion of Corollary 3.3 is true on $M \times[0, T)$ for some constant $C$. From this, Remark 1 and Proposition 2.1 we see that if $T<\infty$ we could then extend $v$ as a solution to (4.12) to $M \times\left[0, T^{\prime}\right)$ for some $T^{\prime}>T$ which contradicts the maximality of 
$T$. Thus we must have $T=\infty$ which establishes the first part of the Theorem.

We now prove the second part of the Theorem on convergence. In [6] a $C^{0}$ estimate was established for (4.12) under the conditions of Theorem 4.2 and the additional assumption that $\Omega=0$. The key difference in this case is that the corresponding metrics $g(t)$ will evolve under the standard Kähler-Ricci flow, and general Kähler-Ricci theory may then be applied. On the other hand, the a priori estimates of the previous section basically ensure that by the same proof as in [6] we obtain the same $C^{0}$ estimate without this additional assumption. Our a priori estimates and Proposition 2.1 then imply the existence of a longtime solution to (4.12) which stays uniformly bounded in the $C^{0}$ norm on $M$ with additional higher order derivative bounds aswell. We state this more precisely in the following

Lemma 4.1. Let $\left(M^{n}, g_{0}\right)$ be as in Theorem 4.2. Then (4.12) has a smooth solution $v$ on $M \times[0, \infty)$ such that for each $0 \leq l \leq 4$, $\left\|\nabla_{0}^{l} v(x, t)\right\|_{g_{0}}$ is bounded by a constant depending only on $l, g_{0}, f_{0}$.

Moreover, given any $4<l<\infty$ and a compact set $S \subset M$ then $\left\|\nabla_{0}^{l} v(x, t)\right\|_{S, g_{0}}$ is bounded by a constant depending only on $S, l, g_{0}, f_{0}$.

Proof. By Proposition 2.1, there exists a solution $v(x, t)$ to (4.12) on $M \times[0, T)$ for some $T>0$ satisfying the properties in the Proposition. Thus $g_{i \bar{\jmath}}(t)=\left(g_{0}\right)_{i \bar{\jmath}}+v_{i \bar{\jmath}}$ is a family of Kähler metrics on $M$ such that for each $t \in[0, T), g(t)$ is equivalent to $g_{0}$. Moreover, by remark 1 we in fact have that for each $t \in[0, T), g(t)$ has bounded curvature. It follows from the proof of Lemma 4 in [6] that we have $\sup _{t \in[0, T)}|v(t)| \leq C$ for some $C$ independent of $T$.

In particular, by Corollary 3.3 we have $\|v\|_{C^{2+\alpha, 1+\alpha / 2}(M \times[0, T))}$ bounded independent of $T$. Thus by considering the pull back of (3.1) in an arbitrary quasi-coordinate and applying a bootstrapping argument, as in the last part of the proof of Proposition [2.1, we conclude that $\|v(t)\|_{C^{4+\alpha}(M)}$ is bounded independent of $t \in[0, T)$ (see Remark 1). Thus by Proposition 2.1 we can extend $v(x, t)$ as a solution to (4.12) past $T$, and we may then assume that $T=\infty$ in the above discussion. This completes the proof of the first part of the Lemma. The proof of the second part of the Lemma follows from iterating the above bootstrapping argument.

Let $v(x, t)$ is a longtime solution to (4.12) as in Lemma4.1. We want investigate the longtime behavior of $w(x, t)=u_{t}(x, t)$.

Lemma 4.2. $w \rightarrow 0$ pointwise on $M$ as $t \rightarrow \infty$. 
Proof. We begin by showing that $\left|\nabla w^{k}\right| \rightarrow 0$ as $t \rightarrow \infty$ for some integer $k \geq 1$. By (15) of [6], if $p=2 k+2$ with $k$ being a large integer, we have

$$
\frac{d}{d t} \int_{M} w^{p} d V_{t} \leq-C \int_{M}\left|\nabla w^{k}\right|^{2} d V_{t}
$$

where $C$ is a positive constant independent of $t$. Let $x_{0} \in M$ suppose there exist $t_{i} \rightarrow \infty$ and $\epsilon>0$ such that $\left|\nabla w^{k}\right|\left(x_{0}, t_{i}\right) \geq \epsilon$. On the other hand, there is a neighborhood $U$ of $x_{0}$, such that

$$
\left|u_{t \alpha \beta}\right|+\left|u_{t t \alpha}\right| \leq C
$$

in $U$, where $\alpha$ etc. denote the indices for the real coordinates. Hence there is $\delta>0$ such that for all $(x, t) \in B_{0}\left(x_{0}\right) \times\left[t_{i}, t_{i}+\delta\right]$ we have $\left|\nabla w^{k}\right| \geq \frac{\epsilon}{2}$. In particular,

$$
\int_{M}\left|\nabla w^{k}\right|^{2} d V_{t} \geq \epsilon^{\prime}
$$

for all $t \in\left[t_{i}, t_{i}+\delta\right]$ for some $\epsilon^{\prime}>0$ independent of $i$. This is impossible because of (4.14). Thus we have established that $\left|\nabla w^{k}\right| \rightarrow 0$ as $t \rightarrow \infty$ for some integer $k \geq 1$. On the other hand, (4.14) shows that the integral $\int_{M} w^{p} d V_{t}$ is uniformly bounded for all $t \geq 0$. Combining these last two facts together with Lemma 4.1, we conclude that $w \rightarrow 0$ pointwise on $M$ as $t \rightarrow \infty$.

We now combine the above lemma's with a Liouville theorem of Yau [22] for $L^{p}$ harmonic functions on complete Riemannian manifolds to give a proof of Theorem 4.2 .

Proof of Theorem 4.2. By Lemma 4.1, given any sequence $t_{i} \rightarrow \infty$ there is a subsequence of $u\left(x, t_{i}\right)$ converging smoothly and uniformly on compact subsets of $M$. To prove Theorem 4.2 it suffices to prove that such a limit is independent of the sequence $t_{i}$ which we now proceed to do.

Suppose $u\left(x, t_{i}\right)$ converges to $u_{1}(x)$ and $u\left(x, s_{i}\right)$ converges to $u_{2}(x)$ smoothly and uniformly on compact subsets of $M$, where $t_{i}, s_{i} \rightarrow \infty$ and $s_{i} \leq t_{i}$ for every $i$. We claim that $v=u_{1}-u_{2}$ satisfies the Laplace equation

$$
\Delta_{h} v=0
$$

on $M$ where $h$ is a complete Kähler metric on $M$ which is equivalent to $g_{0}$ and has all covariant derivatives of its curvature tensor bounded. 
Indeed, by (4.12) we have

$$
\begin{aligned}
f\left(s_{i}\right)-f\left(t_{i}\right) & =\log \operatorname{det}\left(\left(g_{0}\right)_{k \bar{l}}+u_{k \bar{l}}\left(t_{i}\right)\right)-\log \operatorname{det}\left(\left(g_{0}\right)_{k \bar{l}}+u_{k \bar{l}}\left(s_{i}\right)\right) \\
& =\int_{0}^{1} \frac{d}{d s} \log \operatorname{det}\left(\left(g_{0}\right)_{k \bar{l}}+\left(s u\left(t_{i}\right)-(1-s) u\left(s_{i}\right)\right)_{k \bar{l}}\right) d s \\
& =\left(\int_{0}^{1} G^{l \bar{k}}(s) d s\right)\left(u\left(t_{i}\right)-u\left(s_{i}\right)\right)_{l \bar{k}}
\end{aligned}
$$

for each $i$, where $G^{i \bar{\jmath}}(s)$ is the inverse of the Kähler metric $G_{i \bar{\jmath}}(s)=$ $\left.\left(g_{0}\right)_{k \bar{l}}+\left(s u\left(t_{i}\right)-(1-s) u\left(s_{i}\right)\right)_{k \bar{l}}\right)$. It follows from Lemma 4.1 that some subsequence of $\left(\int_{0}^{1} G^{l \bar{k}}(s) d s\right)$ converges smoothly and uniformly on compact subsets of $M$ to a smooth limit $h^{i \bar{\jmath}}$ which is the inverse of a Kähler metric $h$ as above. Our claim follows by taking a limit of (4.17) and using Lemma 4.2. On the other hand, by the proof of

Lemma 3 in [6] we know that $\int_{M}|u(x, t)|^{p} d V_{0} \leq C$ for some $p$ and some $C$ independent of $t$. Thus we have $\int_{M}|v(x)|^{p} d V_{0} \leq C$. It follows from this, (4.16) and the Liouville theorems in 23. that $v=0$ and thus $u_{1}=u_{2}$. This completes the proof of Theorem 4.2.

\section{ApPEndiX}

We begin by explicitly defining the local Hölder norms used in the definitions of the elliptic and parabolic Hölder spaces on $M$ used in $\S 2$ (Also see [10]). Let $\Omega$ be an open set in $\mathbb{R}^{m}$. Let $k \geq 0$ be an integer and $0<\alpha<1$, then the $C^{k+\alpha}$ norm of a function $u$ on $\Omega$ is defined as:

$$
\|u\|_{k+\alpha ; \Omega}=\sum_{|s|=0}^{k} \sup _{\Omega}\left|\partial^{s} u\right|+\sum_{|s|=k} \sup _{x \neq x^{\prime} \in \Omega} \frac{\left|\partial^{s} u(x)-\partial^{s} u\left(x^{\prime}\right)\right|}{\left|x-x^{\prime}\right|^{\alpha}}
$$

where $s$ is a multi-index, and

$$
\partial^{s} u=\frac{\partial^{|s|} u}{\partial x^{s}}
$$

For $T>0$, the $C^{2 k+\alpha, k+\frac{\alpha}{2}}$ norm on $\Omega_{T}=\Omega \times[0, T]$ is defined as:

$$
\begin{aligned}
\|u\|_{k+\alpha, k+\frac{\alpha}{2} ; \Omega_{T}}= & \sum_{|s|+2 r=0}^{2 k} \sup _{\Omega}\left|\partial_{t}^{r} \partial^{s} u\right| \\
& +\sum_{|s|+2 r=2 k} \sup _{(x, t) \neq\left(x^{\prime}, t^{\prime}\right) \in \Omega_{T}} \frac{\left|\partial_{t}^{r} \partial^{s} u(x, t)-\partial_{t}^{r} \partial^{s} u\left(x^{\prime}, t^{\prime}\right)\right|}{\left|x-x^{\prime}\right|^{\alpha}+\left|t-t^{\prime}\right|^{\frac{\alpha}{2}}}
\end{aligned}
$$


where

$$
\partial_{t}^{r} \partial^{s} u=\frac{\partial^{r+|s|} u}{\partial t^{r} \partial x^{s}}
$$

If there is no confusion, we will simply write $\|u\|_{k+\alpha}$ or $\|u\|_{k+\alpha, k+\frac{\alpha}{2}}$.

Now suppose $(M, g)$ has bounded geometry of order $k+\alpha$ with respect to a quasi-coordinate system $\mathcal{F}$ where $k$ is even. Fix some $T>0$ and consider the associated parabolic Hölder norm $\|\cdot\|_{k, \alpha ; \mathcal{F}}$ for functions on $M \times[0, T)$ as in Definition 2.2 where the additional subscript denotes dependence on the quasi-coordinate $\mathcal{F}$. The following lemma basically says that the associated Banach space $C^{k+\alpha, k / 2+\alpha / 2}(M \times[0, T))$ from definition 2.2 is independent of the choice of quasi-coordinate.

Lemma 5.1. Let $\mathcal{G}=\left\{\left(\theta_{p}, V_{p}\right) \mid p \in M\right\}$ be another family of quasicoordinate neighborhoods with data $K_{1}, K_{2}, R$. Then there is a constant $C>0$ such that for all smooth function $f$ on $M \times[0, T]$

$$
\|f\|_{k, \alpha ; \mathcal{G}} \leq C\|f\|_{k, \alpha ; \mathcal{F}}
$$

Proof. We just prove the case that $k=2$. Let $f$ be a smooth function on $M \times[0, T]$. Let $\left(\theta_{p}, V_{p}\right)$ be a quasi-coordinate neighborhood in $\mathcal{G}$. Let $w_{0} \in D(R)$ and let $\theta_{p}\left(w_{0}\right)=q$. Let $\left(\xi_{q}, U_{q}\right)$ be a quasi-coordinate in $\mathcal{F}$ such that $\xi_{q}(0)=q$. By [4, Lemma 3.2] in [4], suppose $D\left(w_{0}, \epsilon\right)=$ $\left\{\left|w-w_{0}\right|<\epsilon\right\} \subset D(R)$, then there is a local biholomorphism $\phi:$ $D\left(w_{0}, \epsilon\right) \rightarrow D(r)$ such that $\phi\left(w_{0}\right)=0$ and $\xi_{q} \circ \phi=\theta_{p}$, provided $\epsilon<\epsilon_{0}$ which depends only on $\mathcal{F}$ and $\mathcal{G}$. Suppose $\phi(w)=z$. Then

$$
\xi_{q}^{*}(f)=\theta_{p}^{*}(f)
$$

and

$$
s_{i \bar{\jmath}} \frac{\partial z_{i}}{\partial w_{k}} \frac{\overline{\partial z_{j}}}{\partial w_{l}} .=h_{k \bar{l}}
$$

on $\theta\left(D\left(w_{0}, \epsilon\right)\right)$ where $h_{k \bar{l}}:=\theta_{q}^{*}(g)\left(\frac{\partial}{\partial w_{k}}, \frac{\partial}{\partial \bar{w}_{l}}\right)$ and $s_{i \bar{\jmath}}:=\xi_{p}^{*}(g)\left(\frac{\partial}{\partial z_{k}}, \frac{\partial}{\partial \bar{z}_{l}}\right)$.

Let $k=l$ and using the fact that $\xi_{q}^{*}(g)$ and $\theta_{p}^{*}(g)$ are uniformly equivalent to the Euclidean metric, we conclude that

$$
\left|\frac{\partial z_{i}}{\partial w_{k}}\right| \leq C
$$

on $D\left(w_{0}, \epsilon\right)$. Now

$$
\frac{\partial \theta_{p}^{*}(f)}{\partial w_{k}}=\frac{\partial \xi_{q}^{*}(f)}{\partial z_{i}} \frac{\partial z_{i}}{\partial w_{k}}
$$

we conclude that on $D\left(w_{0}, \epsilon\right)$

$$
\left|\theta_{p}^{*}(f)\right|+\left|D_{w} \theta_{p}^{*}(f)\right| \leq C\|f\|_{2, \alpha, \mathcal{F}}
$$


It is also easy to see that on $D\left(w_{0}, \epsilon\right)$

$$
\left|D_{t} \theta_{p}^{*}(f)\right|=\left|D_{t} \xi_{q}^{*}(f)\right| \leq C|| f \|_{2, \alpha, \mathcal{F}}
$$

On the other hand,

$$
\begin{aligned}
\frac{\partial}{\partial w_{a}} h_{i \bar{\jmath}}= & \frac{\partial}{\partial z_{b}} s_{k \bar{l}} \frac{\partial z_{b}}{\partial w_{a}} \frac{\partial z_{k}}{\partial w_{i}} \frac{\overline{\partial z_{l}}}{\partial w_{j}} \\
& +s_{k \bar{l}} \frac{\partial^{2} z_{k}}{\partial w_{i} \partial w_{a}} \frac{\overline{\partial z_{l}}}{\partial w_{j}}
\end{aligned}
$$

Consider the vector $v=\frac{\partial}{\partial z_{k}} \frac{\partial^{2} z_{k}}{\partial w_{i} \partial w_{a}}$. Let $v=a_{j} \phi_{*} \frac{\partial}{\partial w_{j}}$.

$$
\xi_{q}^{*}(g)\left(v, \phi_{*} \frac{\partial}{\partial \bar{w}_{c}}\right)=a_{j} h_{j \bar{c}} .
$$

Hence

$$
\begin{aligned}
v & =h^{j \bar{c}} \xi_{q}^{*}(g)\left(v, \phi_{*} \frac{\partial}{\partial \bar{w}_{c}}\right) \phi_{*} \frac{\partial}{\partial w_{j}} \\
& =h^{j \bar{c}} s_{k \bar{l}} \frac{\partial^{2} z_{k}}{\partial w_{i} \partial w_{a}} \frac{\overline{\partial z_{l}}}{\partial w_{c}} \frac{\partial z_{d}}{\partial w_{j}} \frac{\partial}{\partial z_{d}} .
\end{aligned}
$$

So

$$
\begin{aligned}
\frac{\partial^{2} z_{d}}{\partial w_{i} \partial w_{a}} & =h^{j \bar{c}} s_{k \bar{l}} \frac{\partial^{2} z_{k}}{\partial w_{i} \partial w_{a}} \frac{\overline{\partial z_{l}}}{\partial w_{c}} \frac{\partial z_{d}}{\partial w_{j}} \\
& =h^{j \bar{c}}\left(\frac{\partial}{\partial w_{a}} h_{i \bar{\jmath}}-\frac{\partial}{\partial z_{b}} s_{k \bar{l}} \frac{\partial z_{b}}{\partial w_{a}} \frac{\partial z_{k}}{\partial w_{i}} \frac{\overline{\partial z_{l}}}{\partial w_{j}}\right) \frac{\partial z_{d}}{\partial w_{j}}
\end{aligned}
$$

Hence we have

$$
\left|\frac{\partial^{2} z_{d}}{\partial w_{i} \partial w_{a}}\right| \leq C
$$

on $D\left(w_{0}, \epsilon\right)$. In fact, from $(0.3)$ we see that

$$
\left\|\frac{\partial^{3} z_{d}}{\partial w_{i} \partial w_{a} \partial w_{j}}\right\|_{\alpha} \leq C
$$

ion $D\left(w_{0}, \epsilon\right)$ (Note that since $s_{i \bar{\jmath}}(z)$ is $C^{2+\alpha}$ and $z(w)$ is $C^{1}$, it follows that $\frac{\partial^{2}}{\partial z^{2}} s_{i \bar{\jmath}}(z(w))$ is $C^{\alpha}$ with respect to $\left.w\right)$. In particular, it is easy to see that on $D\left(w_{0}, \epsilon\right)$

$$
\left|D_{w}^{2} \theta_{p}^{*}(f)\right| \leq C\|f\|_{2, \alpha, \mathcal{F}} .
$$

Now if $w \in D\left(w_{0}, \epsilon\right)$, then 


$$
\begin{aligned}
\frac{\partial \theta_{p}^{*}(f)}{\partial w_{k} \partial w_{l}}(w) & -\frac{\partial \theta_{p}^{*}(f)}{\partial w_{k} \partial w_{l}}\left(w_{0}\right) \\
= & \frac{\partial \xi_{q}^{*}(f)}{\partial z_{i} \partial z_{j}} \frac{\partial z_{i}}{\partial w_{k}} \frac{\partial z_{j}}{\partial w_{l}}(w)+\frac{\partial \xi_{q}^{*}(f)}{\partial z_{i}} \frac{\partial^{2} z_{i}}{\partial w_{k} \partial w_{l}}(w) \\
& -\frac{\partial \xi_{q}^{*}(f)}{\partial z_{i} \partial z_{j}} \frac{\partial z_{i}}{\partial w_{k}} \frac{\partial z_{j}}{\partial w_{l}}\left(w_{0}\right)-\frac{\partial \xi_{q}^{*}(f)}{\partial z_{i}} \frac{\partial^{2} z_{i}}{\partial w_{k} \partial w_{l}}\left(w_{0}\right) .
\end{aligned}
$$

Since $\left|z-z_{0}\right| \leq C\left|w-w_{0}\right|$, we can conclude that

$$
\frac{\left|\frac{\partial \theta_{p}^{*}(f)}{\partial w_{k} \partial w_{l}}(w)-\frac{\partial \theta_{p}^{*}(f)}{\partial w_{k} \partial w_{l}}\left(w_{0}\right)\right|}{\left(\left|w-w_{0}\right|^{2}+\left|t-t_{0}\right|\right)^{\frac{\alpha}{2}}} \leq C\|f\|_{2, \alpha, \mathcal{F}}
$$

for $w \in D\left(w_{0}, \epsilon\right)$. One can also obtain the Hölder estimate for $\partial_{t} \theta_{p}^{*}(f)$. This completes the proof of the Lemma.

\section{REFERENCES}

1. Cao, Huai-Dong, Deformation of Kähler metrics to Kahler Einstein metrics on compact Kahler manifolds, Invent. Math. 81 (1985), 359-372.

2. Chau, A., Convergence of the Kähler Ricci flow on non-compact Kähler Manifolds, J. Differential Geom. 66 (2004), 211-232.

3. A. Chau, J. Chen, W. He, Lagrangian Mean Curvature flow for entire Lipschitz graphs, arXiv:0902.3300.

4. Chau, A., Tam, L.F., Non-negatively curved Kähler manifolds with average quadratic curvature decay, Comm. Anal. Geom. 15 (2007), no. 1, 121-146,

5. Chau, A., Tam, L.F., and Yu, C., Pseudolocality for the Ricci flow and applications, to appear in Canadian Journal of Math.

6. Chau, A., Tam, L.F., A $C^{0}$-estimate for the parabolic Monge-Ampère equation on complete non-compact Kähler manifolds.

7. Cheng, S.Y. and Yau, S.T., On the existence of a complete Kahler Einstein metric on non-compact complex manifolds and the regularity of Feffermans equation, Comm. Pure App. Math. 33 (1980), 507-544.

8. Friedman, A., Partial differential equations of parabolic type, Prentice Hall, 1964.

9. Hamilton, R.S., Three-manifolds with positive Ricci curvature, J. Differential Geom. 17 (1982), 255-306.

10. Krylov, N.V., Lectures on elliptic and parabolic equations in Hölder spaces, Graduate Studies in Mathematics, 12. American Mathematical Society, Providence, RI, 1996.

11. Lott J. and Zhang Z., Ricci flow on Quasiprojective manifolds., arXiv 0906.4496v2

12. Ni, L. and Tam, L.-F., Plurisubharmonic functions and the Kähler-Ricci flow, Amer. J. Math. 125 (2003), no. 3, 623-654. 
13. Ni, L. and Tam, L.-F., Plurisubharmonic functions and the structure of complete Kähler manifolds with nonnegative curvature J. Differential Geom. 64 (2003), no. 3, 457-524,

14. Shi, Wan-Xiong, Ricci deformation of the metric on complete non-compact Riemannian manifolds, J. Differential Geom. 30 (1989), 303-394.

15. Shi, Wan-Xiong, Ricci Flow and the uniformization on complete non compact Kähler manifolds, J. of Differential Geometry. 45 (1997), no. 1, 94-220.

16. Tam, Luen-fai, Exhaustion function on complete manifolds, to appear in Proceedings of the The Ninth Pacific Rim Geometry Conference, 2008, Taipei.

17. Tian, G. and Yau S.T., Existence of Kähler Einstein metrics on complete Kahler manifolds and their applications to algebraic geometry, in "Mathematical Aspects of String Theory", edited by S.T. Yau, World Scientific, 1986.

18. Tian, G. and Yau S.T., Complete Kähler manifolds with zero Ricci curvature. I., J. Amer. Math. Soc. 3(1990), no.3, 579-609.

19. Tian, G. and Yau S.T., Complete Kahler manifolds with zero Ricci curvature. II., Invent. Math. 106(1990), no.1, 27-60.

20. Tian, G. and Zhang, Z. On the Kähler-Ricci flow on projective manifolds of general type, Chinese Ann. Math. Ser. B 27 (2006), no. 2, 179-192

21. Tsuji, H. Existence and degeneration of $\mathrm{K}$ ähler-Einstein metrics on minimal algebraic varieties of general type, Math. Ann. 281 (1988), 123-133.

22. Yau S.T., On the Ricci curvature of a compact Kähler manifold and the complex Monge-Ampère equation. I, Communications on Pure and Applied Mathematics 31 (1978), 339-441.

23. Yau S.T., Some function-theoretic properties of complete Riemannian manifolds and their applications to geometry, Indiana Univ Math J. 25, (1976), 659-670.

Department of Mathematics, The University of British Columbia, Room 121, 1984 Mathematics Road, Vancouver, B.C., Canada V6T 1 Z2 E-mail address: chau@math.ubc.ca

The Institute of Mathematical Sciences and Department of Mathematics, The Chinese University of Hong Kong, Shatin, Hong Kong, CHINA.

E-mail address: lftam@math.cuhk.edu.hk 